\title{
Fe(II)-induced Reduction of labelled Endoperoxides. NMR degradation studies on G3 Factor and its methyl Ether.
}

Christiane André-Barrès, ${ }^{* a}$ Anne-Lise Bottalla, ${ }^{a}$ Stéphane Massou, ${ }^{\mathrm{b}}$ Chantal Zedde, ${ }^{\mathrm{a}}$ Liliane Gorrichon. ${ }^{a}$

${ }^{a}$ Laboratoire de Synthèse et de Physicochimie de Molécules d'Intérêt Biologique, UMR CNRS 5068, Université Paul-Sabatier, 118 route de Narbonne, 31062 Toulouse France.

b Service Commun de RMN, Université Paul Sabatier, 118 route de Narbonne, F-31062 Toulouse cedex 04, France

Fax: 0561558245

E-mail: candre@,chimie.ups-tlse.fr

Procedure for $\mathbf{6}$ and $\mathbf{1 1}$ synthesis S2

General procedure for endoperoxide reduction.

NMR spectrum and determination of purity of compound $\mathbf{6 a}$ S5

NMR spectrum of compound $\mathbf{1 1}$ .S6

Raw intermediate mixtures NMR analysis and chromatograms. S7-8

Raw mixture NMR analysis and chromatogram after completion.

NMR spectra of compound 7

NMR spectra of compound $\mathbf{8}$ S15-17

NMR spectra of compound 9 S20-21

NMR spectra of compound $\mathbf{1 0}$ S $22-25$

NMR spectra of compound 12 S $26-28$

Determination of purity of compounds 7-12 by HPLC:

Chromatograms obtained after separation of compounds 7 on Hyperprep C18 $250 \times 20$ column, $\mathrm{H}_{2} \mathrm{O} / \mathrm{CH}_{3} \mathrm{OH}: 7 / 3,15 \mathrm{ml} / \mathrm{mn}$, detection at $230 \mathrm{~nm}$

Chromatograms obtained after separation of compounds 8, 9, 10 on Hyperprep C18 250 x 20 column, $\mathrm{H}_{2} \mathrm{O} / \mathrm{CH}_{3} \mathrm{CN}: 1 / 1,7 \mathrm{ml} / \mathrm{mn}$, detection at $230 \mathrm{~nm}$ S30 
1-methoxy-4,4,8,8,10,10 hexamethyl-2,3 dioxabicyclo [4.4.0] dec-5-ene-7,9-dione 6 : To a stirred solution of $5(35 \mathrm{mg}, 0.13 \mathrm{mmol})$ in THF $(3 \mathrm{~mL})$ under argon at $-78^{\circ} \mathrm{C}$ was added slowly $\mathrm{n}$-BuLi $(82 \mu \mathrm{L}, 0.13 \mathrm{mmol})$ and the mixture allowed to warm up to $-20^{\circ} \mathrm{C}$. After 15 min TfOMe $(24.6 \mathrm{mg}, 0.15 \mathrm{mmol})$ was added at $-20^{\circ} \mathrm{C}$. The reaction was stirred for $30 \mathrm{~min}$. and was then extracted with ether, the extract was dried $\left(\mathrm{MgSO}_{4}\right)$ and concentrated to give 6 as an oil. Purification by silica gel chromatography (eluent petroleum ether/ethyl acetate 9/1) gives $6(26 \mathrm{mg}, 70 \%) . \delta_{\mathrm{H}}\left(400 \mathrm{MHz} ; \mathrm{CDCl}_{3} ; \mathrm{Me}_{4} \mathrm{Si}\right) 7.35(\mathrm{~s}, 1 \mathrm{H}, \mathrm{C}-\mathrm{CH}), 3.44\left(\mathrm{~s}, 3 \mathrm{H}, \mathrm{OCH}_{3}\right)$, $1.45,1.35,1.32,1.28,1.27,1.03\left(6 \mathrm{~s}, 18 \mathrm{H}, 6 \times \mathrm{CH}_{3}\right)$ ppm. $\delta_{\mathrm{C}}\left(100.62 \mathrm{MHz} ; \mathrm{CDCl}_{3} ; \mathrm{Me}_{4} \mathrm{Si}\right)$ 210.5 (C9), 198.9 (C7), 145.7 (C5), 128.2 (C6), 100.3 (C1), 78.8 (C4), $54.7\left(\mathrm{O}-\mathrm{CH}_{3}\right), 53.1$ (C10), 25.9, 24.7, 23.8, 23.5, 21.6, $15.8\left(6 \times \mathrm{CH}_{3}\right)$ ppm. MS (DCI/NH$) ; m / z(\%): 300$ $\left[\mathrm{MNH}_{4}^{+}\right](100), 283[\mathrm{M}+1]^{+}(13), 210(67)$.

\section{1-fluoro-4,4,8,8,10,10-hexamethyl-2,3-dioxabicyclo[4.4.0]dec-5-ene-7,9-dione, 11}

Compound 5 (1eq., 0,38mmol, 101,7mg) in dichloromethane solution $(2,5 \mathrm{~mL})$ is cooled at $78^{\circ} \mathrm{C}$. Diethylaminosulfur trifluoride (DAST) $(1,5$ eq., $0,57 \mathrm{mmol}, 75 \mu \mathrm{L})$ is then added slowly. After two hours at $-78^{\circ} \mathrm{C}$, the mixture is warmed up to $-40^{\circ} \mathrm{C}$, and kept at this temperature for 4 hours. The reaction mixture is quenched by adding $\mathrm{NaHCO}_{3}$, then extracted with dichloromethane. The organic phase is dried on $\mathrm{MgSO}_{4}$ then evapored. After purification on preparative thin layer chromatography (EP/AcEt 85/15) compound $\mathbf{1 1}$ is obtained with $73 \%$ yield. $\mathrm{MS}\left(\mathrm{DCI} / \mathrm{NH}_{3}\right): m / z(\%)=268\left[\mathrm{MNH}_{4}\right]^{+}(100) ; 285\left[\mathrm{MN}_{2} \mathrm{H}_{7}\right]^{+}(2)$

HRMS (CI/NH $)_{3} \mathrm{C}_{14} \mathrm{H}_{19} \mathrm{O}_{4} \mathrm{~F}:[\mathrm{MH}]^{+}$Calculated/found 271.1346/271.13475.

$\delta_{\mathrm{H}}\left(400 \mathrm{MHz} ; \mathrm{CDCl}_{3} ; \mathrm{Me}_{4} \mathrm{Si}\right) 1.06,1.36,1.38,1.40,1.51\left(6 \mathrm{~s}, 18 \mathrm{H}, 6 \mathrm{CH}_{3}\right.$ en position 11,12 , $13,14,15,16), 7.35\left(\mathrm{~s}, 1 \mathrm{H},=\mathrm{CH}\right.$ on 5 -position). $\delta_{\mathrm{C}}\left(100.62 \mathrm{MHz} ; \mathrm{CDCl}_{3} ; \mathrm{Me}_{4} \mathrm{Si}\right) 15.2,21.2$, 23.3, 23.4, 23.6, 26.1 (6 $\mathrm{CH}_{3}$, on 11, 12, 13, 14, 15, 16 position), $51.8\left(\mathrm{C}_{10}\right), 55.4\left(\mathrm{C}_{8}\right), 79.3$ $\left(\mathrm{C}_{4}\right), 111.3\left(\mathrm{C}_{1}\right), 126.7\left(\mathrm{C}_{6}\right), 145.7\left(\mathrm{~d},{ }^{3} J_{\mathrm{C}-\mathrm{F}}=4,5 \mathrm{~Hz},=C \mathrm{H}\right.$ on 5 position $), 196.5\left(\mathrm{C}_{7}\right), 209.4$ $\left(\mathrm{C}_{9}\right) . \delta_{\mathrm{F}}\left(188.1 \mathrm{MHz} ; \mathrm{CDCl}_{3} ; \mathrm{CF}_{3} \mathrm{COOH}\right):-22,38(\mathrm{~s}, 1 \mathrm{~F})$.

$\operatorname{Rf}($ AcEt / EP : 2 / 8) : 0,59 


\section{General procedure for endoperoxide reduction}

To endoperoxide $(0.12 \mathrm{mmoles})$ dissolved in $1 \mathrm{~mL}$ of degassed acetonitrile, $\mathrm{FeSO}_{4}(31 \mathrm{mg}$, $0.12 \mathrm{mmoles}$ ) first dissolved in $1 \mathrm{~mL}$ of degassed water is added. After $24 \mathrm{~h}, \mathrm{CH}_{3} \mathrm{CN}$ is evaporated, and the water lyophilised. Crude mixture is passed through Chelex resin to get rid of the iron.

\section{Reduction of compound $\mathbf{6}$}

After purification on Hyperprep C18 column (250 x 20) with water/methanol: 40/60, $15 \mathrm{ml} / \mathrm{mn}$, three major compounds $\mathbf{8 , 9}, \mathbf{1 0}$ were obtained in $71 \%$ yield $(1 / 1 / 1)$.

$8 \mathrm{~m} / \mathrm{z}\left(\mathrm{DCI} / \mathrm{NH}_{3}\right) 242\left(\mathrm{MNH}_{4}^{+}, 80 \%\right) 225\left(\mathrm{MH}^{+}, 15 \%\right) 210\left(\mathrm{MH}-\mathrm{CH}_{3}, 100 \%\right) ; v_{\max }(\mathrm{KBr}) /$ $\mathrm{cm}^{-1}$ 2980, 2939, 2873, 1763, 1725, 1682, 1610, 1463, 1381, 1290, 1251, 1189, 1150, 1035. $\delta_{\mathrm{H}}\left(400 \mathrm{MHz} ; \mathrm{CDCl}_{3} ; \mathrm{Me}_{4} \mathrm{Si}\right) 1.36(6 \mathrm{H}, \mathrm{s}, 10$ and $11-\mathrm{Me}), 1.53(6 \mathrm{H}, \mathrm{s}, 8$ and 9-Me), $3.85(3 \mathrm{H}$, s, OMe) $6.81(1 \mathrm{H}, \mathrm{s}, 3-\mathrm{H}) \delta_{\mathrm{C}}\left(100.62 \mathrm{MHz} ; \mathrm{CDCl}_{3} ; \mathrm{Me}_{4} \mathrm{Si}\right) 23.5$ (C10-11), 25.8 (C8-9), 46.0 (C7), 53.0 (C12), 58.0 (C5), 130.0 (C3), 152.0 (C2), 166.0 (C1), 201.6 (C4), 212.5 (C6). $8 \mathbf{a}$ $\delta_{\mathrm{C}} 53.0(\mathrm{C} 12) \mathbf{8 b} / \mathbf{c} \delta_{\mathrm{C}} 166.0(\mathrm{C} 1), 201.6(\mathrm{C} 4)$

$9 \mathrm{~m} / \mathrm{z}\left(\mathrm{DCI} / \mathrm{CH}_{4}\right) 251\left(\mathrm{MH}^{+}, 100 \%\right) ; v_{\max }(\mathrm{KBr}) / \mathrm{cm}^{-1} 3087,2982,2938,2874,1779,1763$, 1708, 1670, 1632, 1616, 1463, 1382, 1310, 1292, 1262, 1189, 1139, 1088, 1051.

$\delta_{\mathrm{H}}\left(400 \mathrm{MHz} ; \mathrm{CDCl}_{3} ; \mathrm{Me}_{4} \mathrm{Si}\right) 1.49(12 \mathrm{H}, \mathrm{s}, 4 \mathrm{Me}) 1.90\left(3 \mathrm{H}, \mathrm{d},{ }^{4} J_{H H} 1.46 \mathrm{~Hz}, 11-\mathrm{Me}\right) 5.56(1 \mathrm{H}$, s, $\left.10-\mathrm{H}_{\mathrm{b}}\right) 5.69\left(1 \mathrm{H}, \mathrm{q},{ }^{4} J_{H H} 1.46 \mathrm{~Hz} \quad 10-\mathrm{H}_{\mathrm{a}}\right) 7.97(1 \mathrm{H}, \mathrm{s}, 4-\mathrm{H})$

$\delta_{\mathrm{C}}\left(100.62 \mathrm{MHz} ; \mathrm{CDl}_{3} ; \mathrm{Me}_{4} \mathrm{Si}\right) 19.5$ (C11), 23.9 (C12-13), 26.0 (C14-15), 59.0 (C7), 84.5 (C3), 124.5 (C10), 129.3 (C5), 145.0 (C9), 166.8 (C4), 168.0 (C1), 195.0 (C6), 201.0 (C8). 9b/c $\delta_{\mathrm{C}} 168.0(\mathrm{C} 1) 195.0(\mathrm{C} 6)$

$10 \mathrm{~m} / \mathrm{z}\left(\mathrm{DCI} / \mathrm{CH}_{4}\right) 253\left(100 \%, \mathrm{MH}^{+}\right) ; v_{\max }(\mathrm{KBr}) / \mathrm{cm}^{-1} 3096,2980,2940,2875,1759,1746$, $1689,1617,1467,1383,1366,1298,1187,1155,1143,1094,1020 ; \delta_{\mathrm{H}}\left(400 \mathrm{MHz} ; \mathrm{CDCl}_{3} ;\right.$ $\left.\mathrm{Me}_{4} \mathrm{Si}\right) 1.12\left(3 \mathrm{H}, \mathrm{d},{ }^{3} J_{\mathrm{HH}} 6.85 \mathrm{~Hz}, 10-11 \mathrm{Me}\right) 1.48(6 \mathrm{H}, \mathrm{s}, 12-13 \mathrm{Me}) 1.51(6 \mathrm{H}, \mathrm{s}, 14-15 \mathrm{Me})$ $3.12\left(1 \mathrm{H}, \mathrm{sept},{ }^{3} J_{\mathrm{HH}} 6.85 \mathrm{~Hz}, 9-\mathrm{H}\right), 8.00(1 \mathrm{H}, \mathrm{s}, 4-\mathrm{H}) . \delta_{\mathrm{C}}\left(100.62 \mathrm{MHz} ; \mathrm{CDCl}_{3} ; \mathrm{Me}_{4} \mathrm{Si}\right) 20.2$ 
(C10-11), 23.0 (C12-13), 26.0 (C14-15), 37.0 (C9), 62.0 (C7), 84.5 (C3), 131.0 (C5), 166.9 (C4), 168.2 (C1), 194.5 (C6), 217.0 (C8). 10b/c $\delta_{\mathrm{C}} 168.2$ (C1), 194.5 (C6).

Reduction of compound $\mathbf{1 1}$

After purification on silicagel with pentane/ethyl acetate: 90/10, a major product 12 was obtained in $60 \%$ yield from 11.

$12 \mathrm{~m} / \mathrm{z}\left(\mathrm{DCI} / \mathrm{NH}_{3}\right) 268\left(\mathrm{MNH}_{4}^{+}\right)$100\%;

$\delta_{\mathrm{H}}\left(400 \mathrm{MHz} ; \mathrm{CDCl}_{3} ; \mathrm{Me}_{4} \mathrm{Si}\right) 1.40(6 \mathrm{H}, \mathrm{s}, 2 \mathrm{Me}, \mathrm{C} 10-11), 1.57$ (6H, s, 2Me, C12-13), 1.64 $(6 \mathrm{H}, \mathrm{s}, 2 \mathrm{Me}, \mathrm{C} 14-15)$

$\delta_{\mathrm{C}}\left(100.62 \mathrm{MHz} ; \mathrm{CDCl}_{3} ; \mathrm{Me}_{4} \mathrm{Si}\right) 23.4$ (C10-11), 24.5 (C12-13), 25.3 (C14-15), 44.3 (C6), 59.3 (C8), 144.8 (C5), 153.4 (C4), 168.8 (C1), 196.1 (C9), 212.5(C7) 
CA78

$6 a$
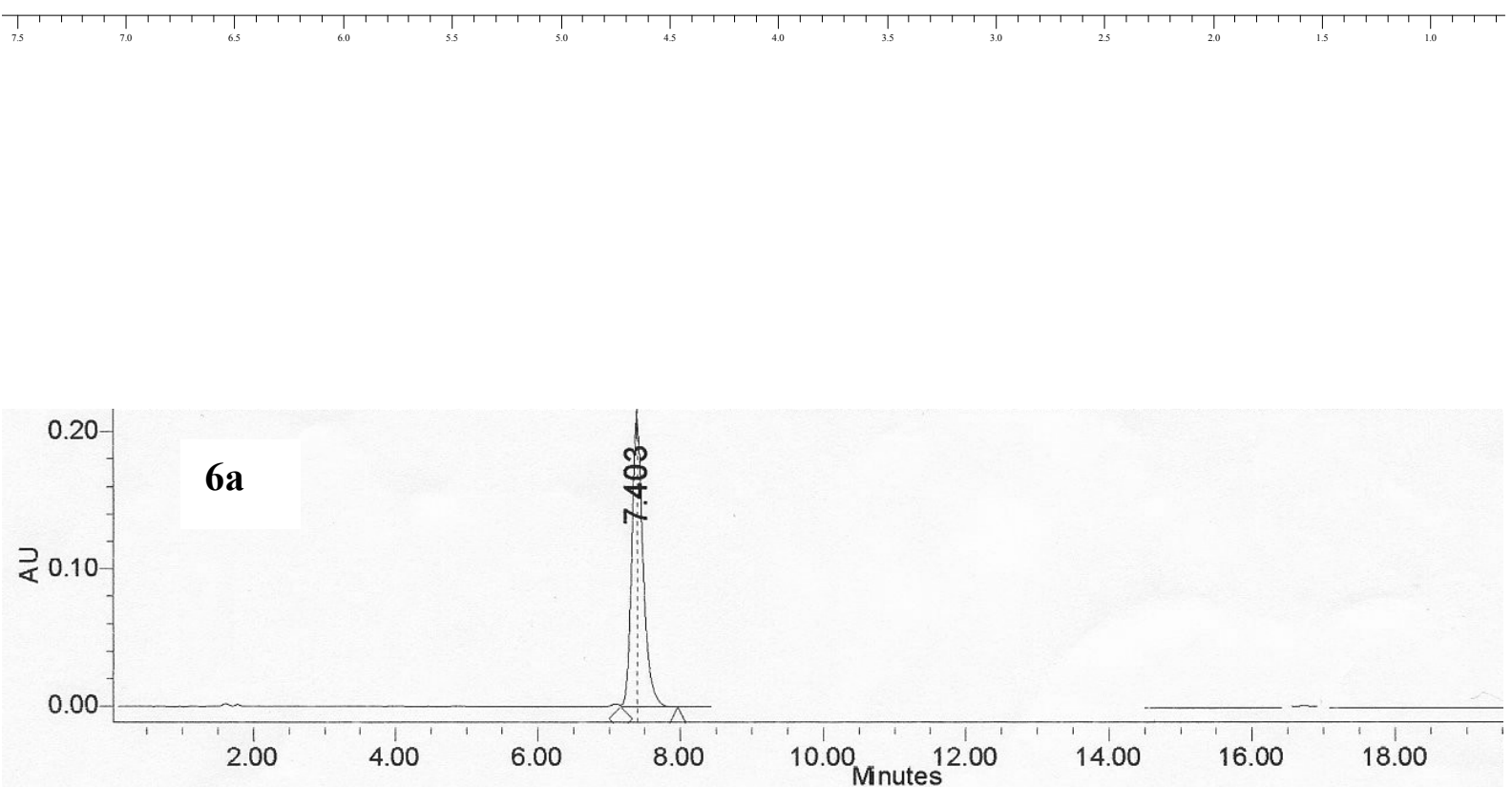


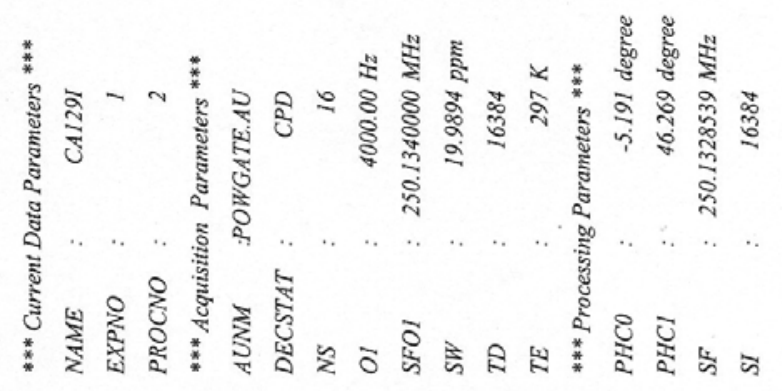

11

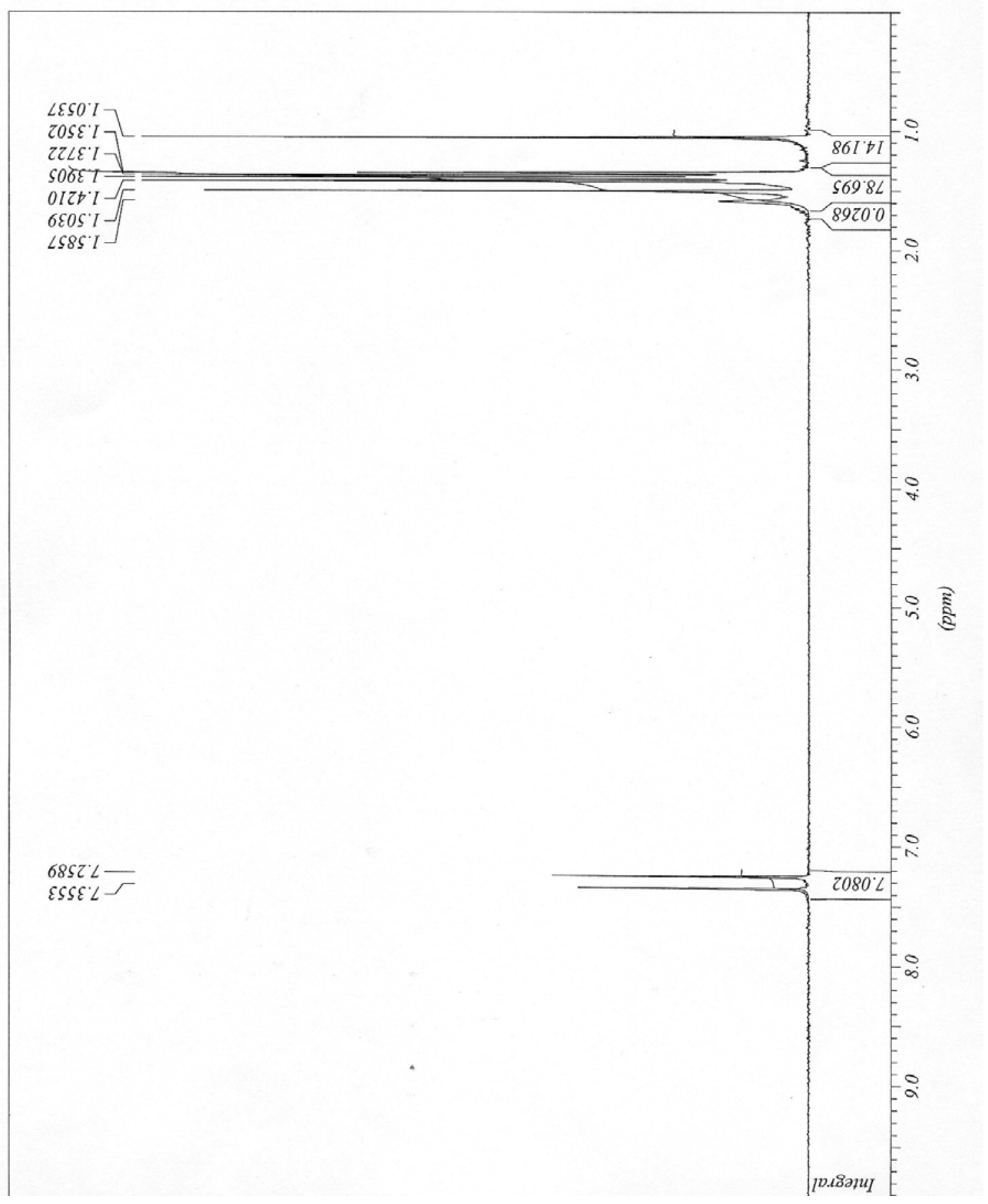


CA95 BRUT

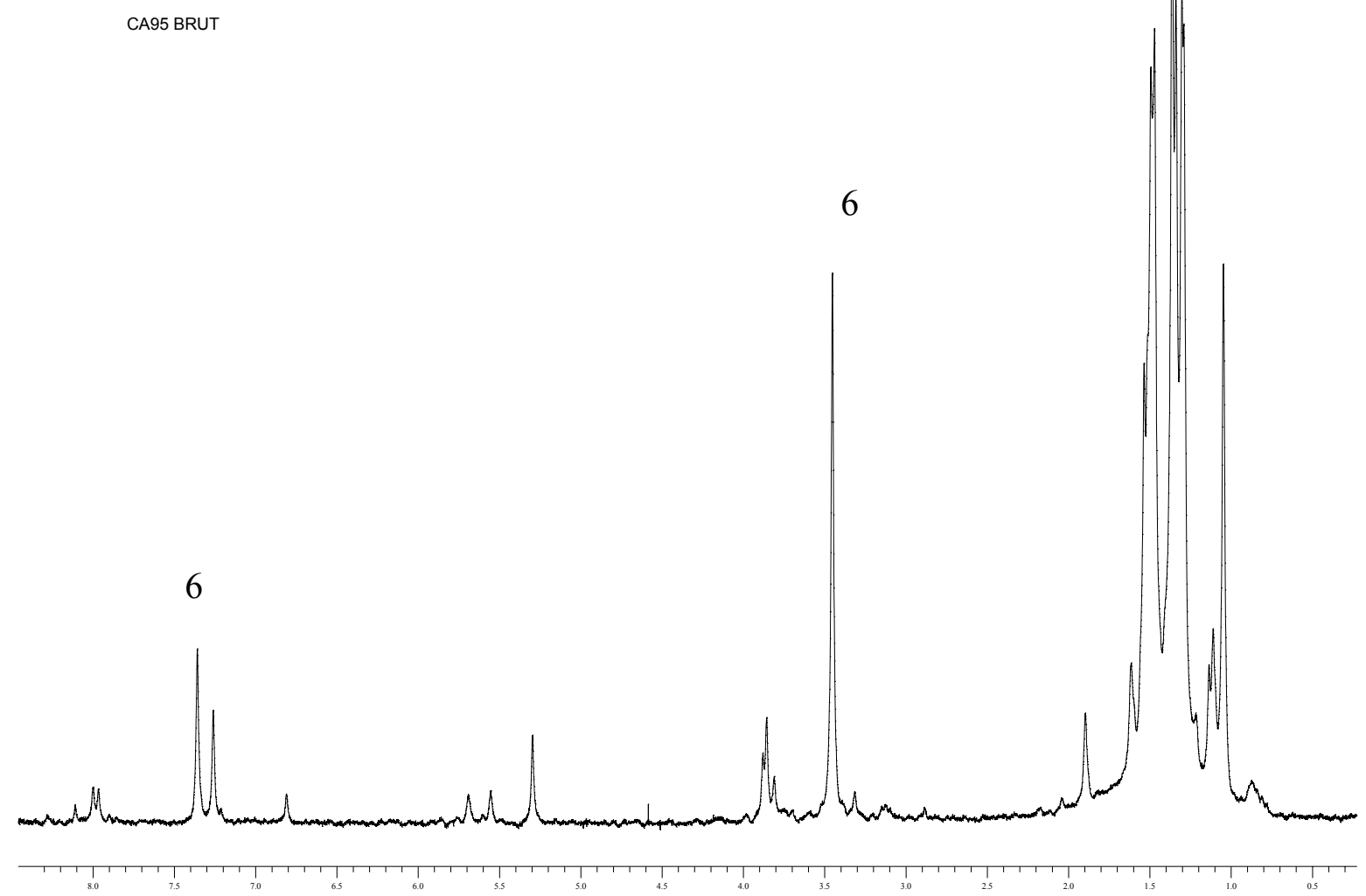

CA113

$6 a$

$6 a$

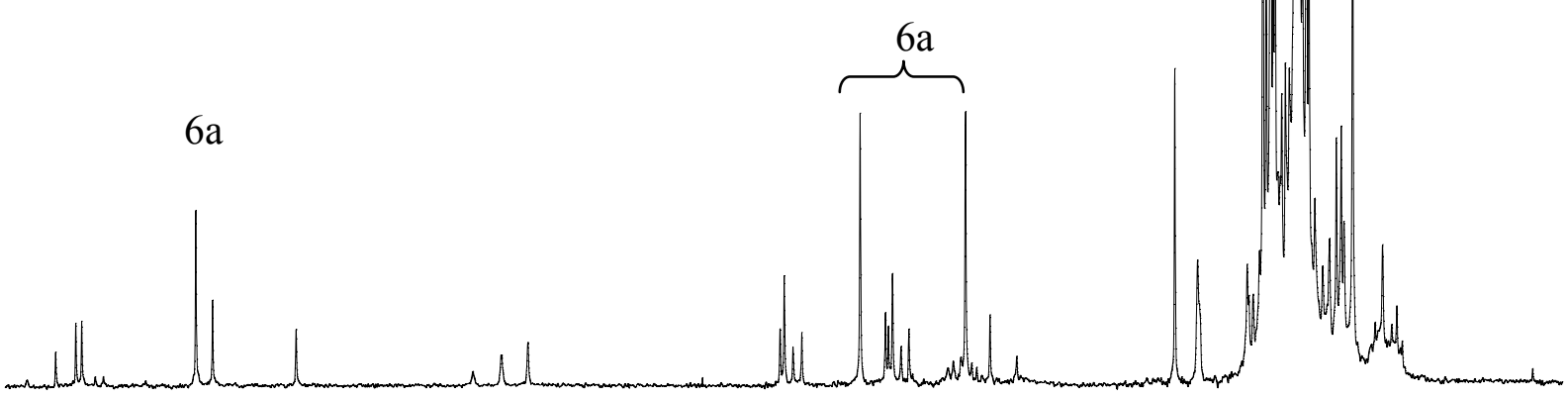

Two typical NMR analysis of uncompleted reduction of $\mathbf{6}$ and $\mathbf{6 a}$ endoperoxides (12h) 


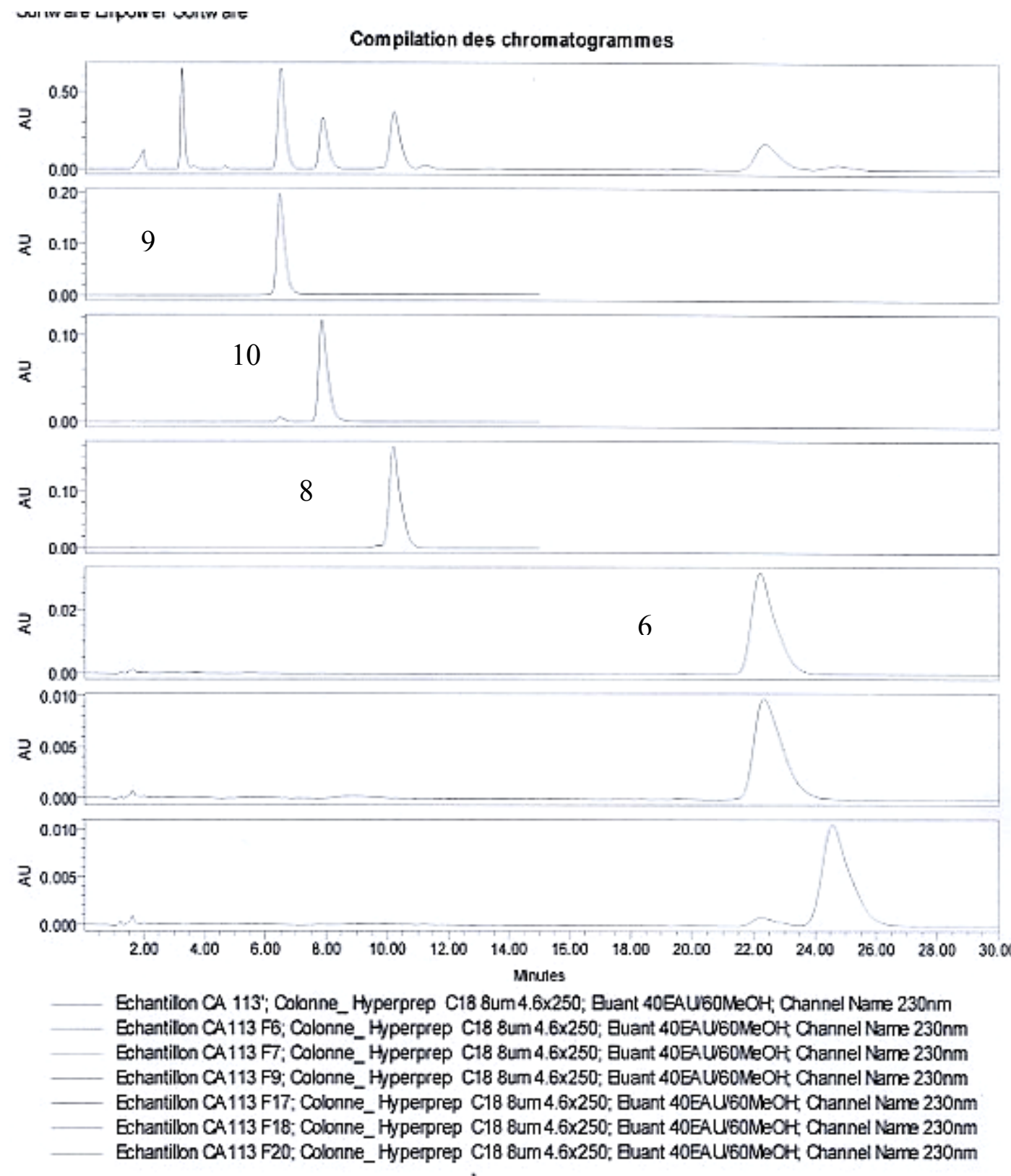

Chromatogram showing the presence of starting material $\mathbf{6}$, together with ending compounds 8, 9, 10 . 

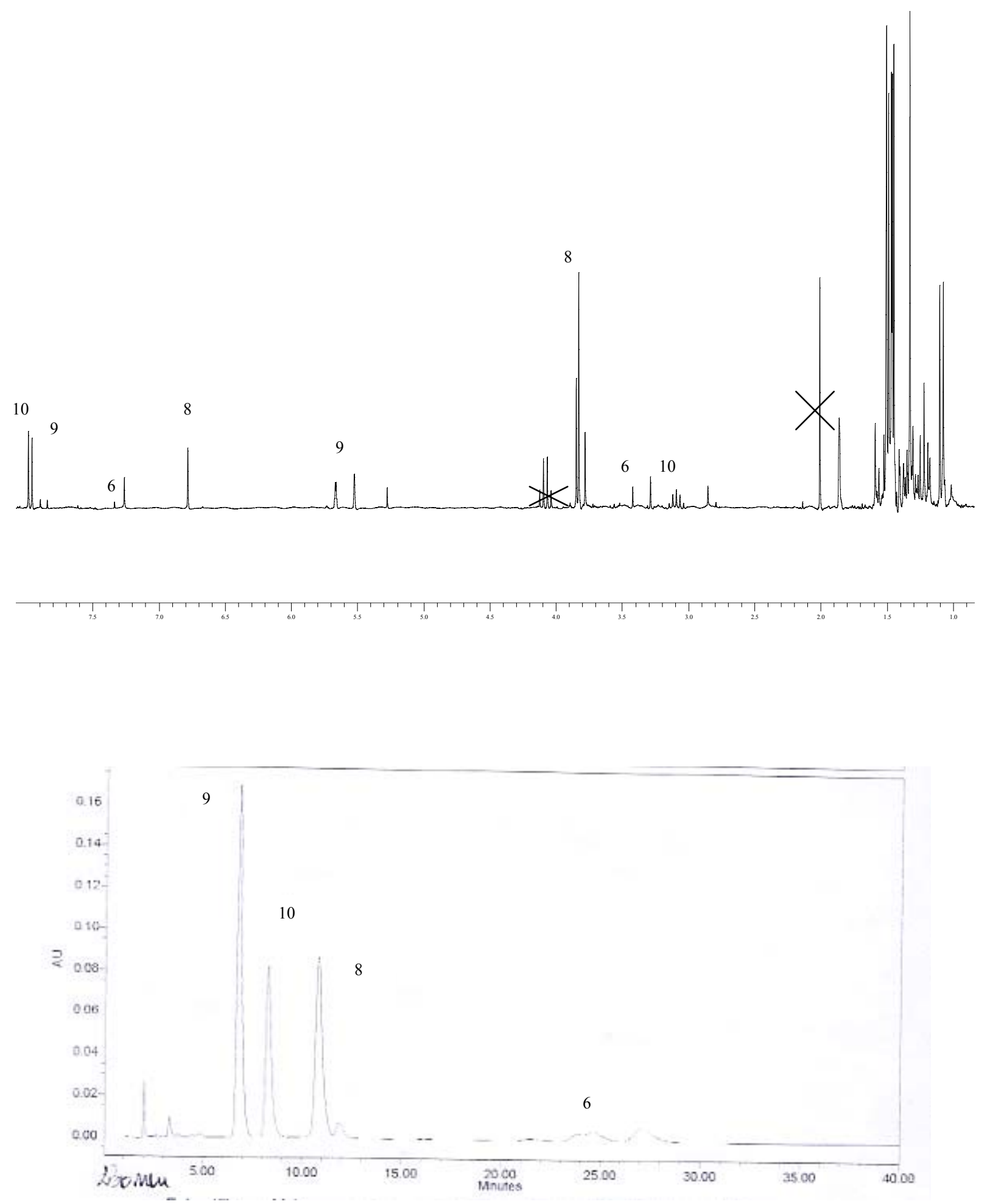

Raw mixture NMR analysis and chromatogram after 24hours. 
$80 \varepsilon^{\prime}$ '

ELS'L

$0^{I}$

r

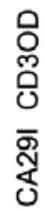

$897^{\circ} 9$

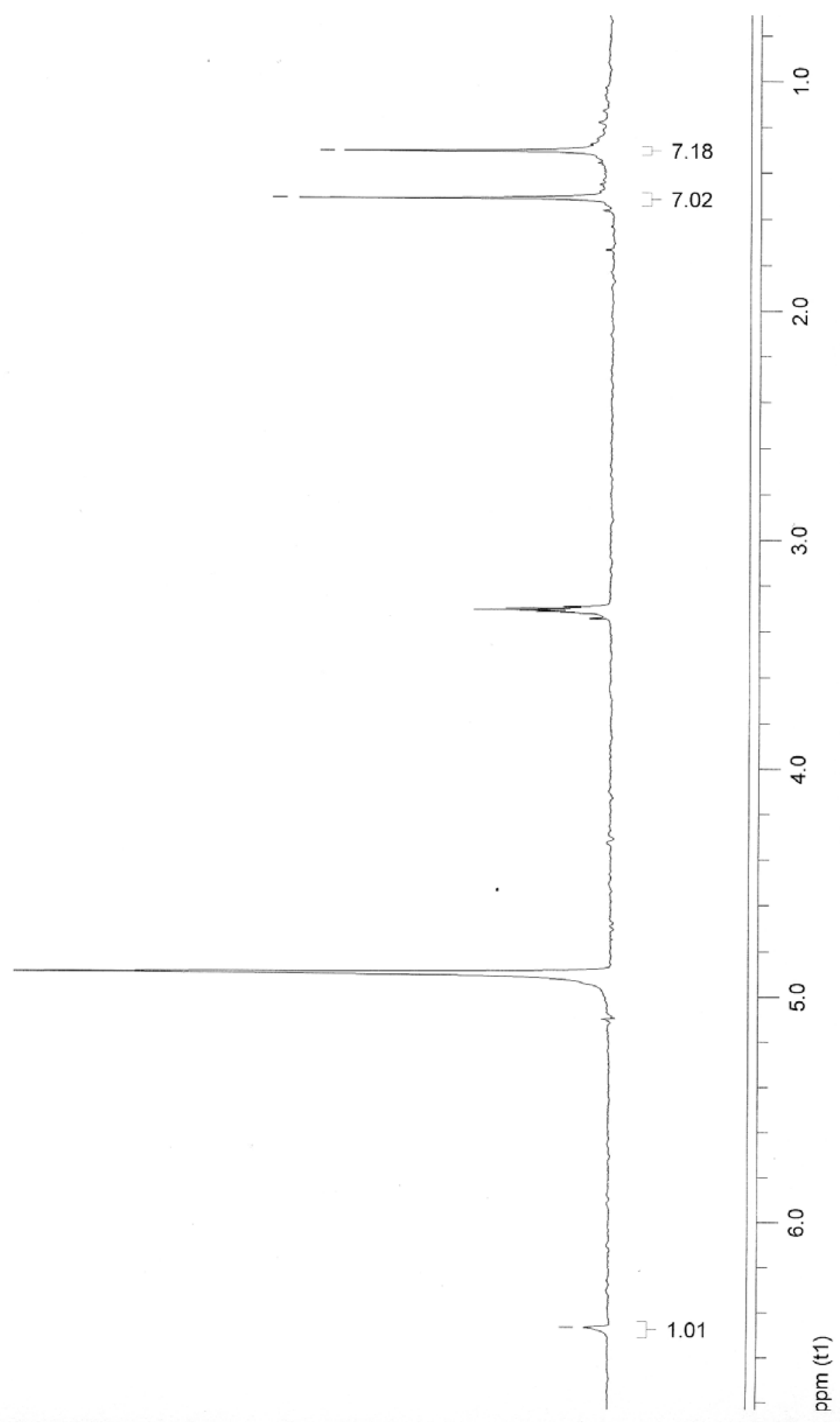

$7{ }^{1} \mathrm{H}$ NMR (250MHz, CD 3 OD) 
-

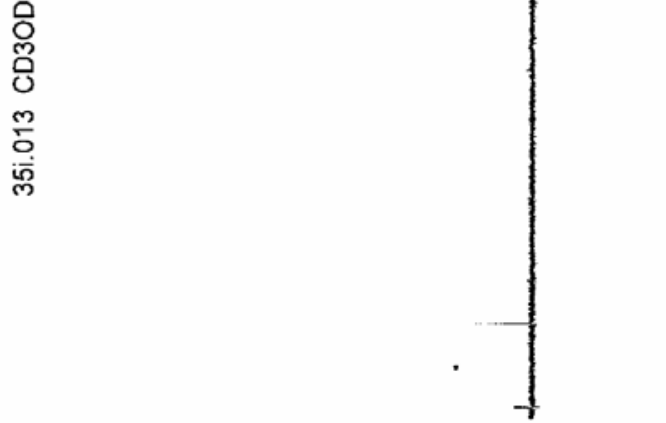

$7{ }^{13} \mathrm{C}$ NMR (50.3MHz, $\left.\mathrm{CD}_{3} \mathrm{OD}\right)$ 


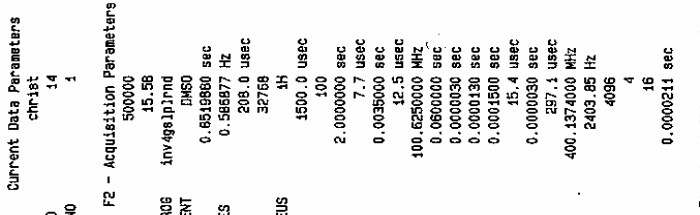

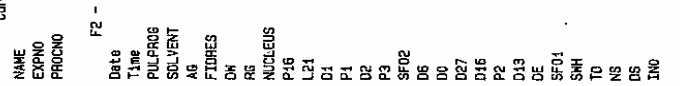

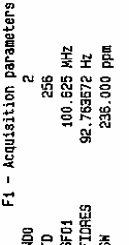

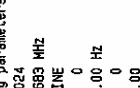

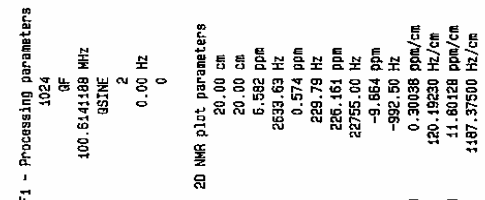
i

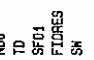

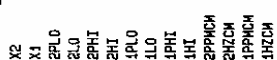

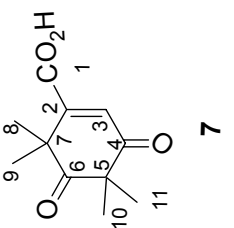

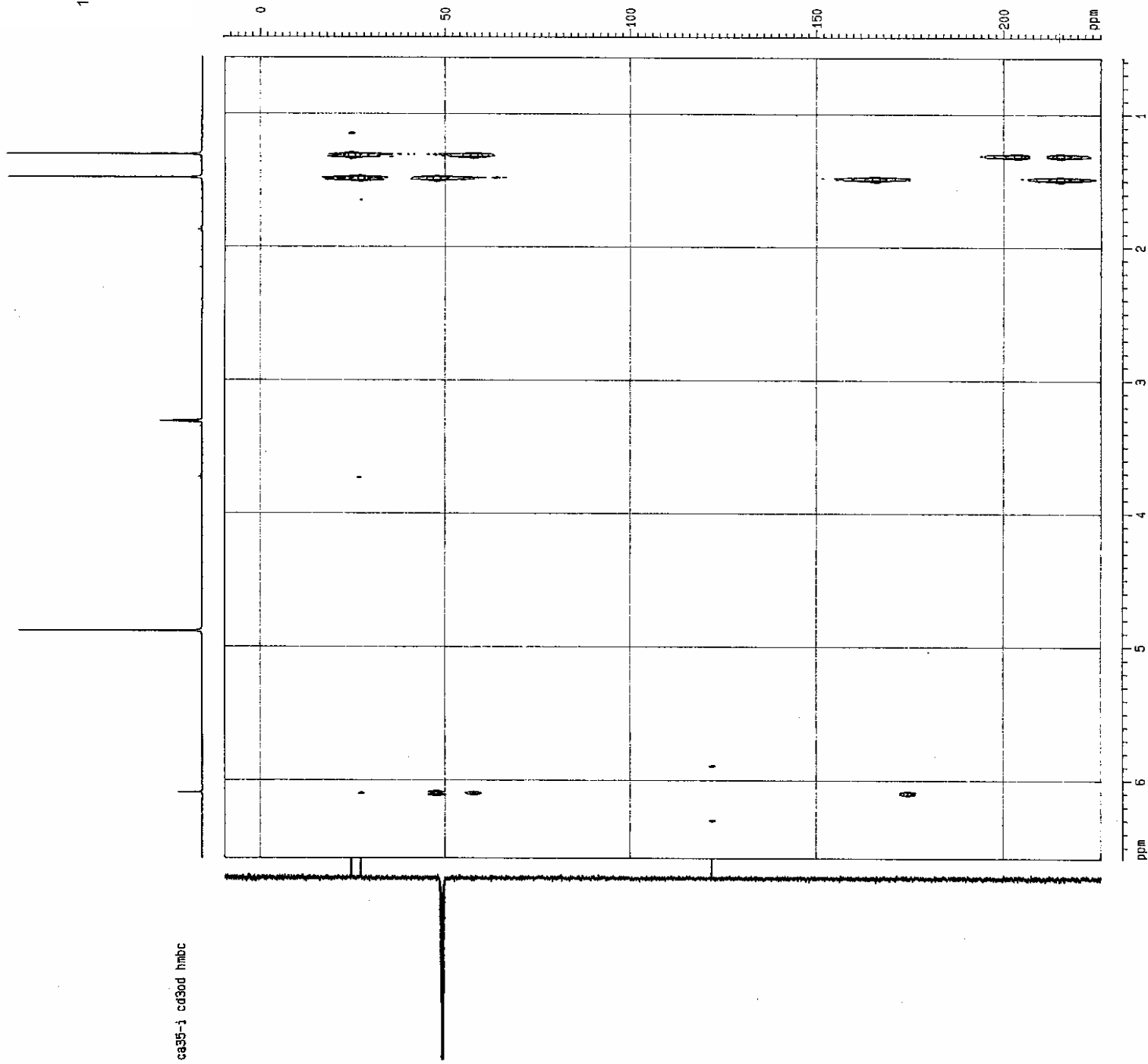

$7 \mathrm{HMBC}\left(400 \mathrm{MHz}, \mathrm{CD}_{3} \mathrm{OD}\right)$ 

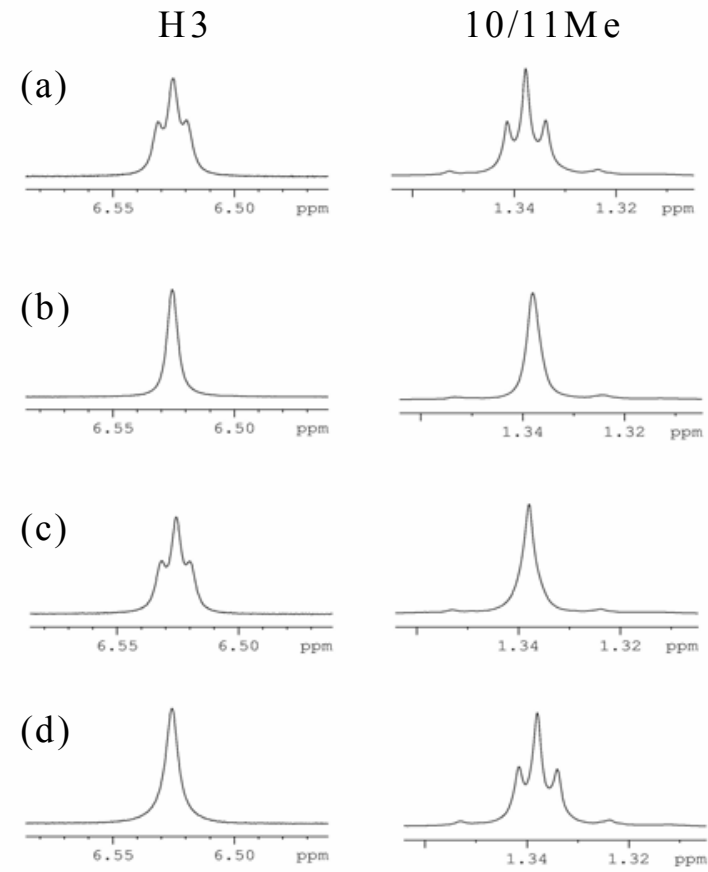

${ }^{13} \mathrm{C}$ decoupling experiments for $7 \mathbf{b} / \mathbf{c}$ : (a) ${ }^{1} \mathrm{H}$ spectrum, (b) ${ }^{13} \mathrm{C}$-decoupled ${ }^{1} \mathrm{H}$ spectrum, (c) ${ }^{13} \mathrm{C}-4$ decoupled ${ }^{1} \mathrm{H}$ spectrum, (d) ${ }^{13} \mathrm{C}-1$ decoupled ${ }^{1} \mathrm{H}$ spectrum. 

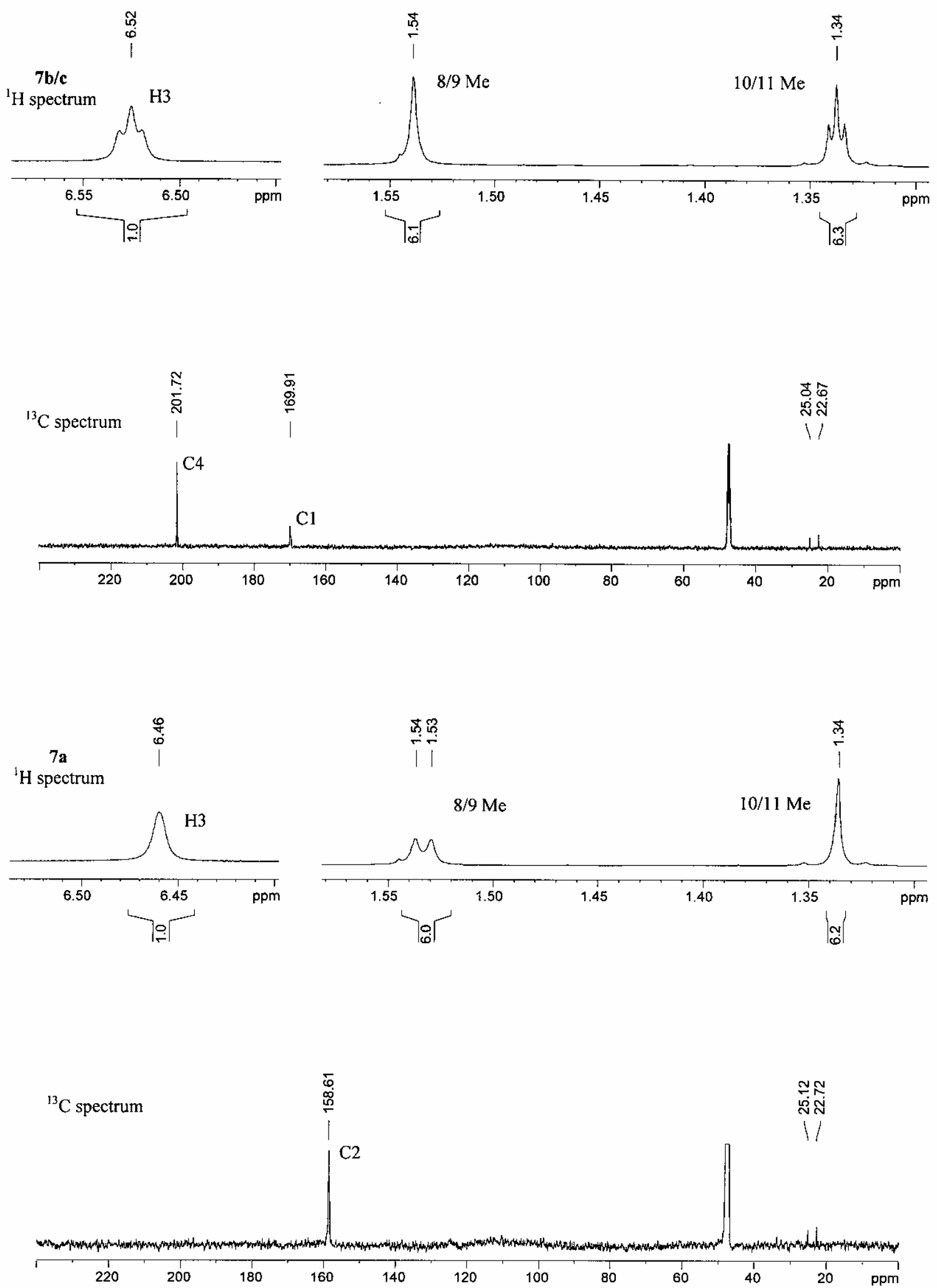

${ }^{1} \mathrm{H}$ and ${ }^{13} \mathrm{C}$ spectra of $\mathbf{7 a}, \mathbf{7 b} / \mathbf{c}\left(500 \mathrm{MHz}, 125 \mathrm{MHz}\right.$ respectively; $\left.\mathrm{CDCl}_{3}\right)$ 


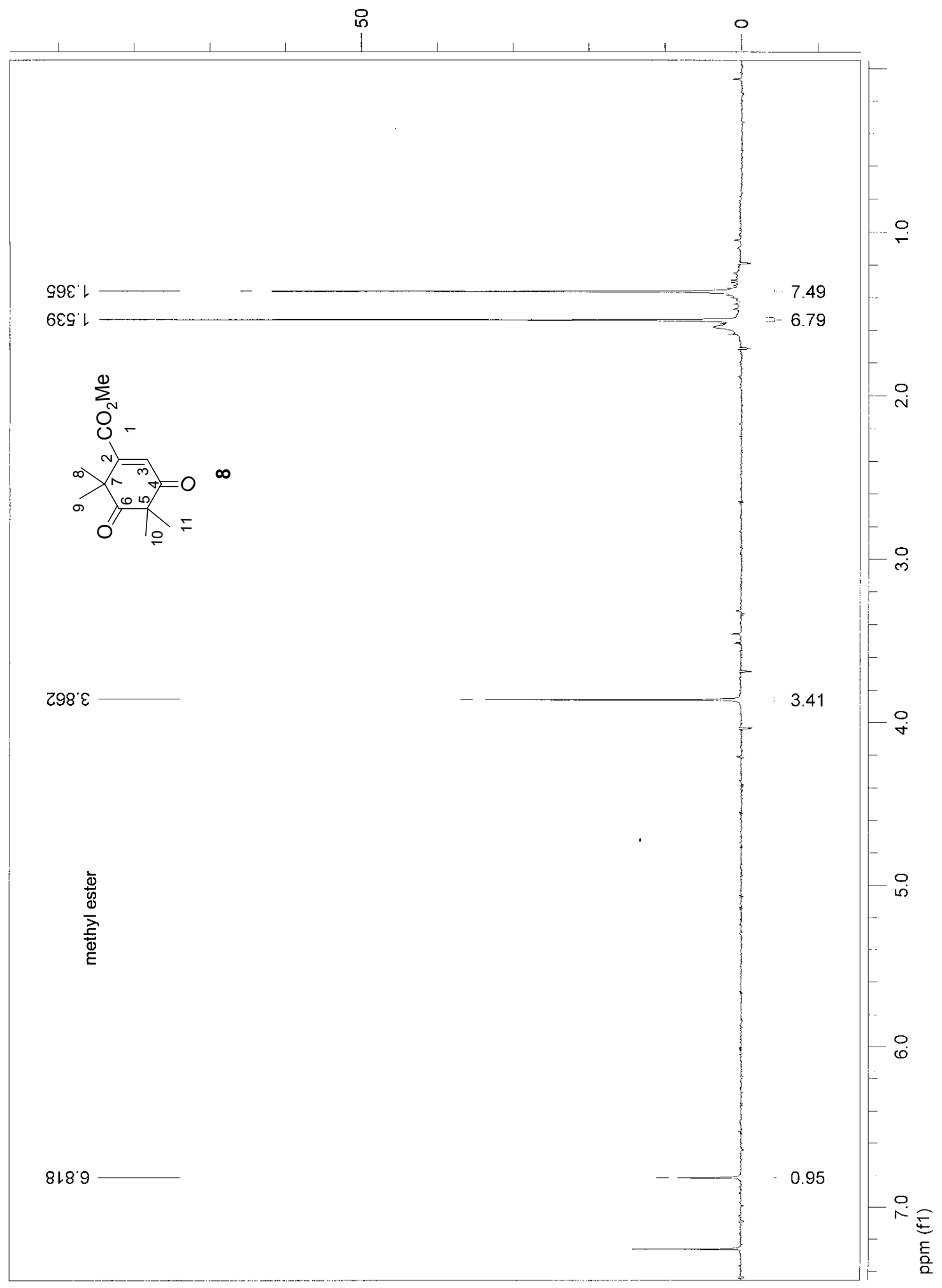

$8{ }^{1} \mathrm{H}$ NMR $\left(250 \mathrm{MHz}, \mathrm{CDCl}_{3}\right)$ 


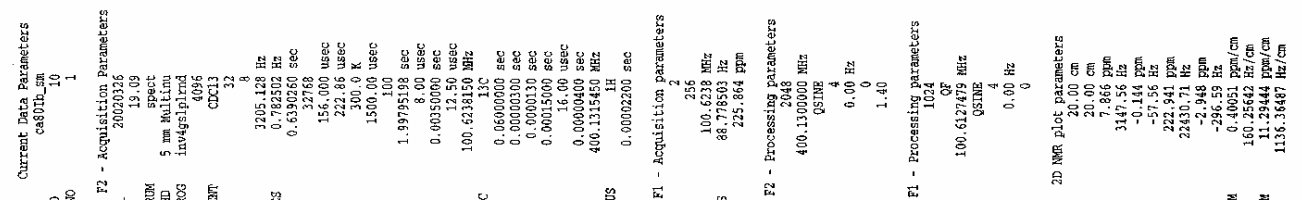

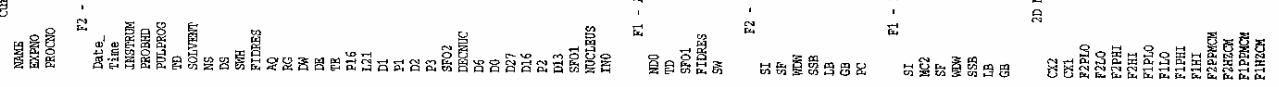

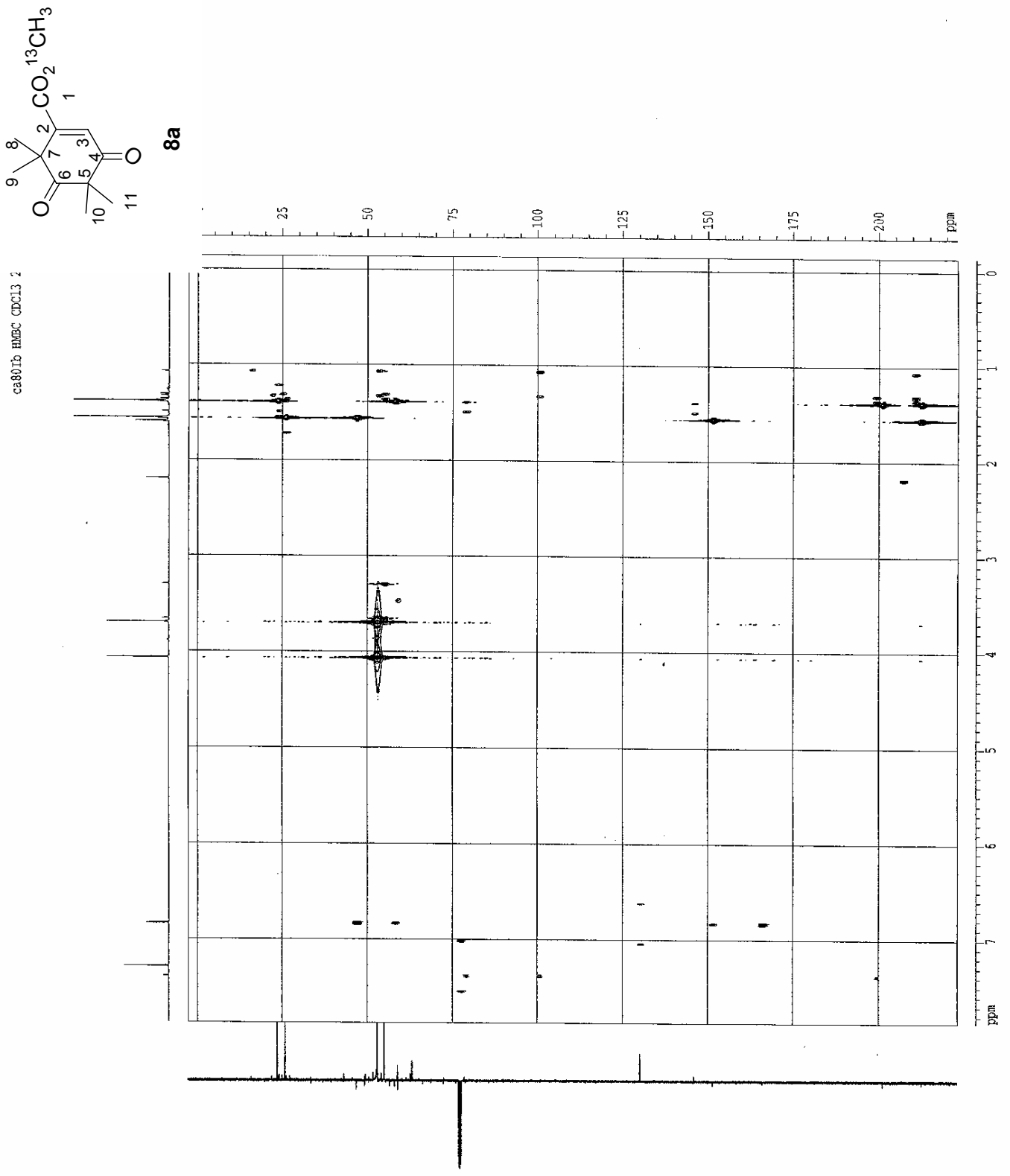

8a $\mathrm{HMBC}\left(400 \mathrm{MHz}, \mathrm{CDCl}_{3}\right)$ 


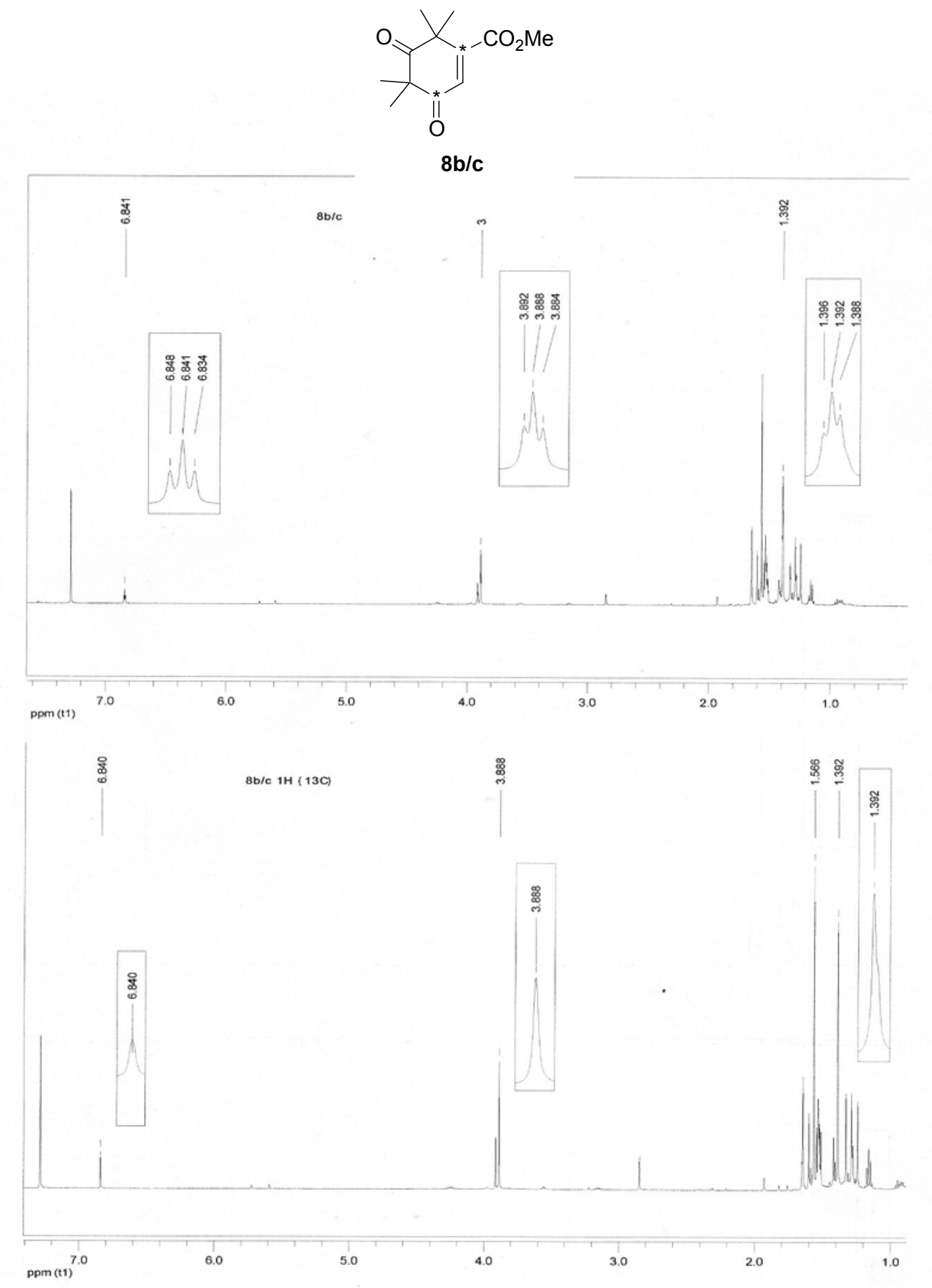

$\mathbf{8 b} / \mathbf{c}{ }^{1} \mathrm{H}$ NMR and $\mathbf{8 b} / \mathbf{c}^{1} \mathrm{H}\left\{{ }^{13} \mathrm{C}\right\}\left(500 \mathrm{MHz}, \mathrm{CDCl}_{3}\right)$ 


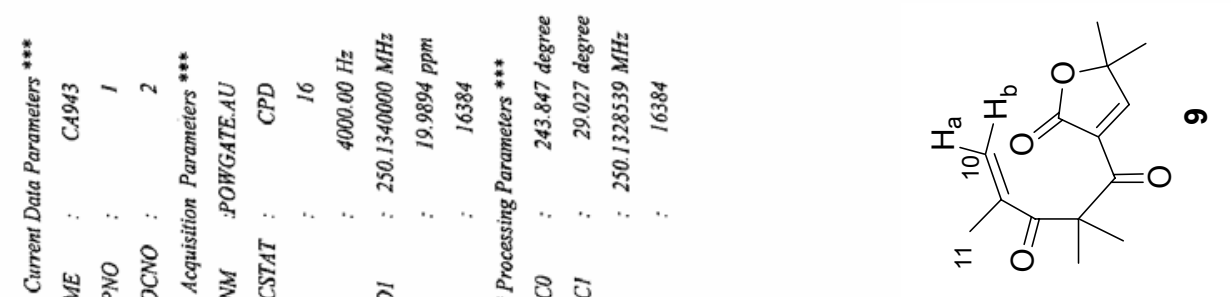

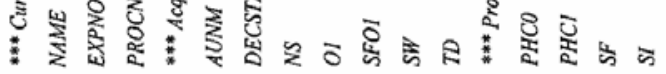

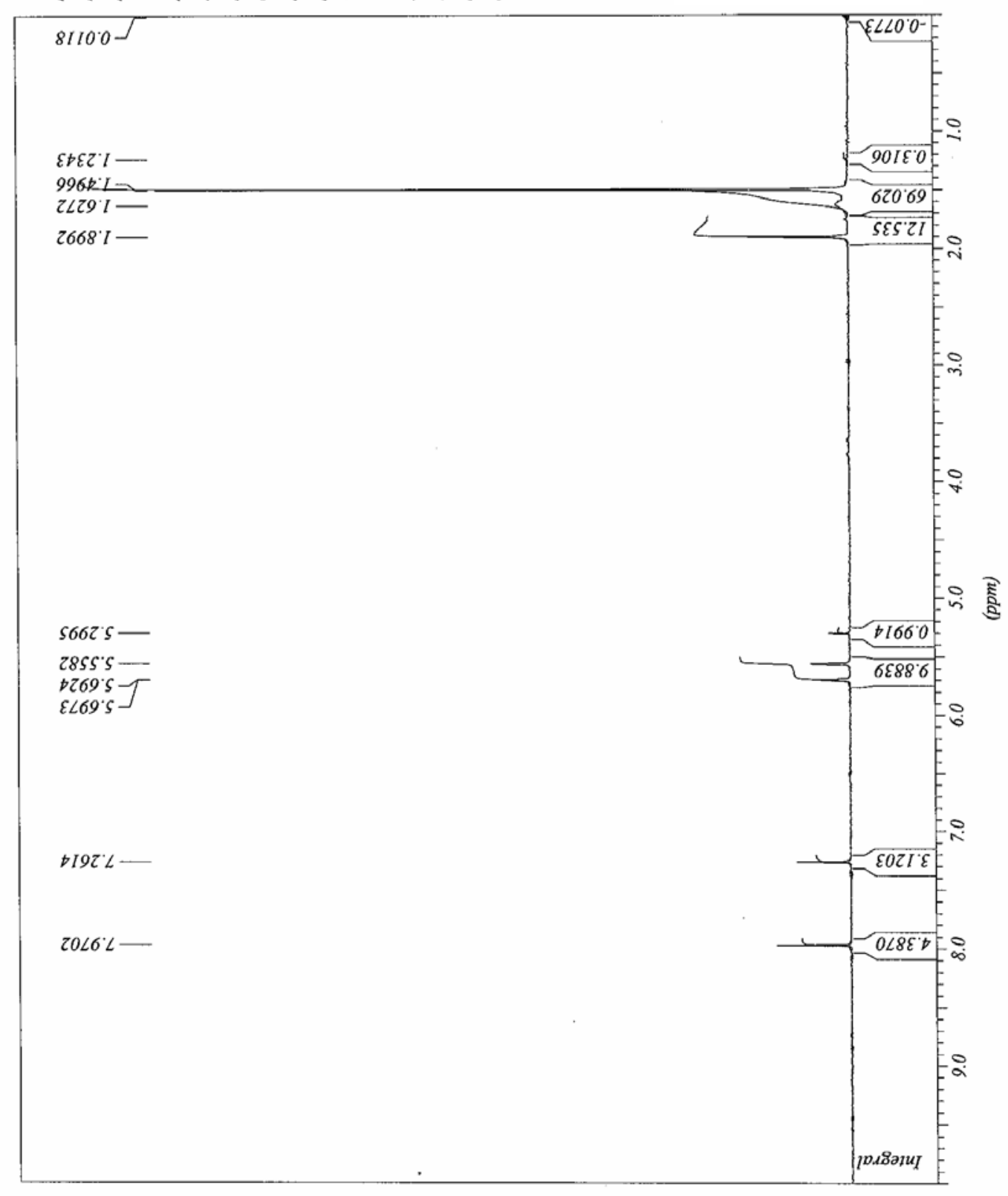

$9{ }^{1} \mathrm{H} \mathrm{NMR}\left(250 \mathrm{MHz}, \mathrm{CDCl}_{3}\right)$ 


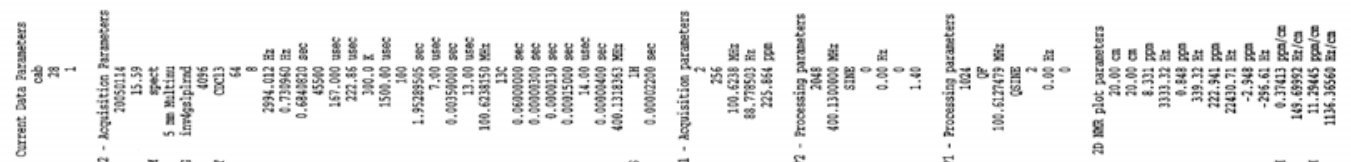

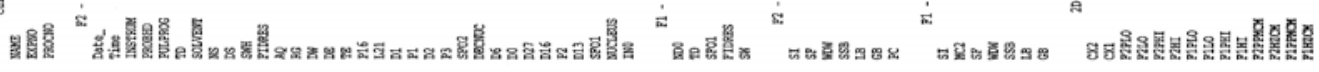
$\prod_{0}^{1}=0$

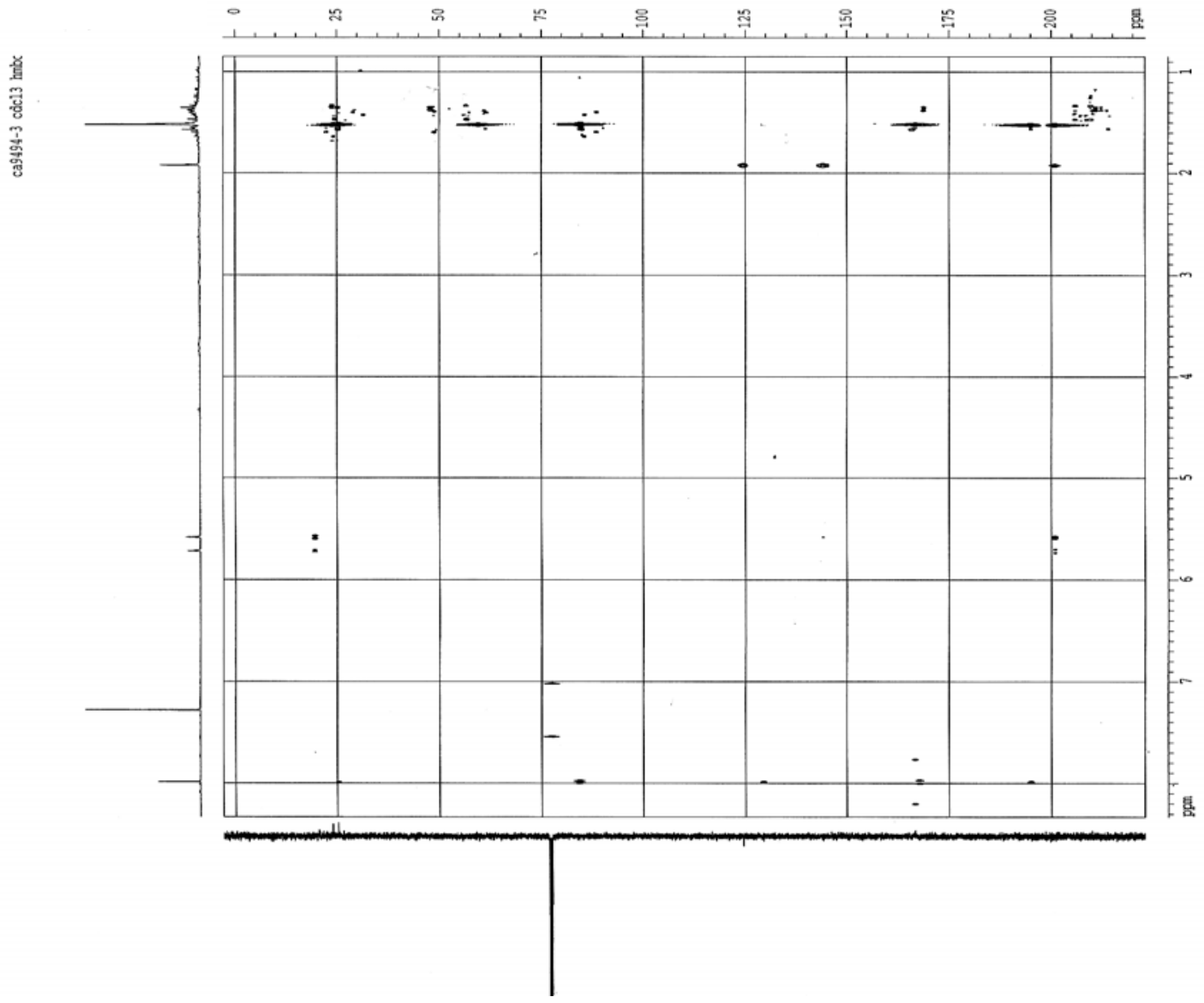

$9 \mathrm{HMBC}\left(400 \mathrm{MHz}, \mathrm{CDCl}_{3}\right)$ 
9b/c $\mathrm{HMBC}\left(500 \mathrm{MHz}, \mathrm{CDCl}_{3}\right)$

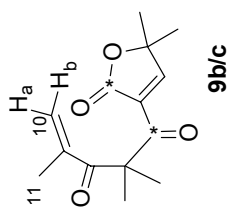

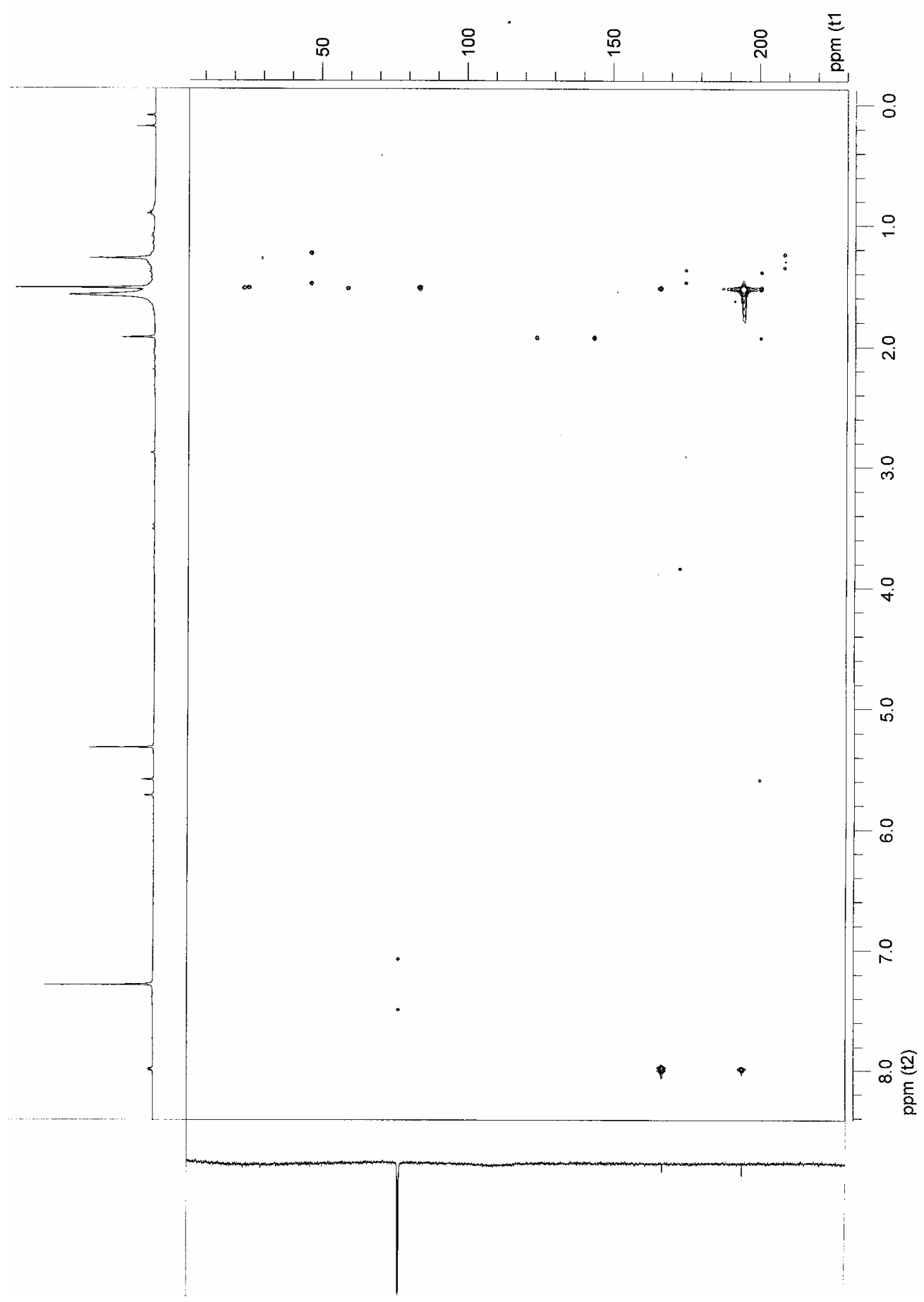



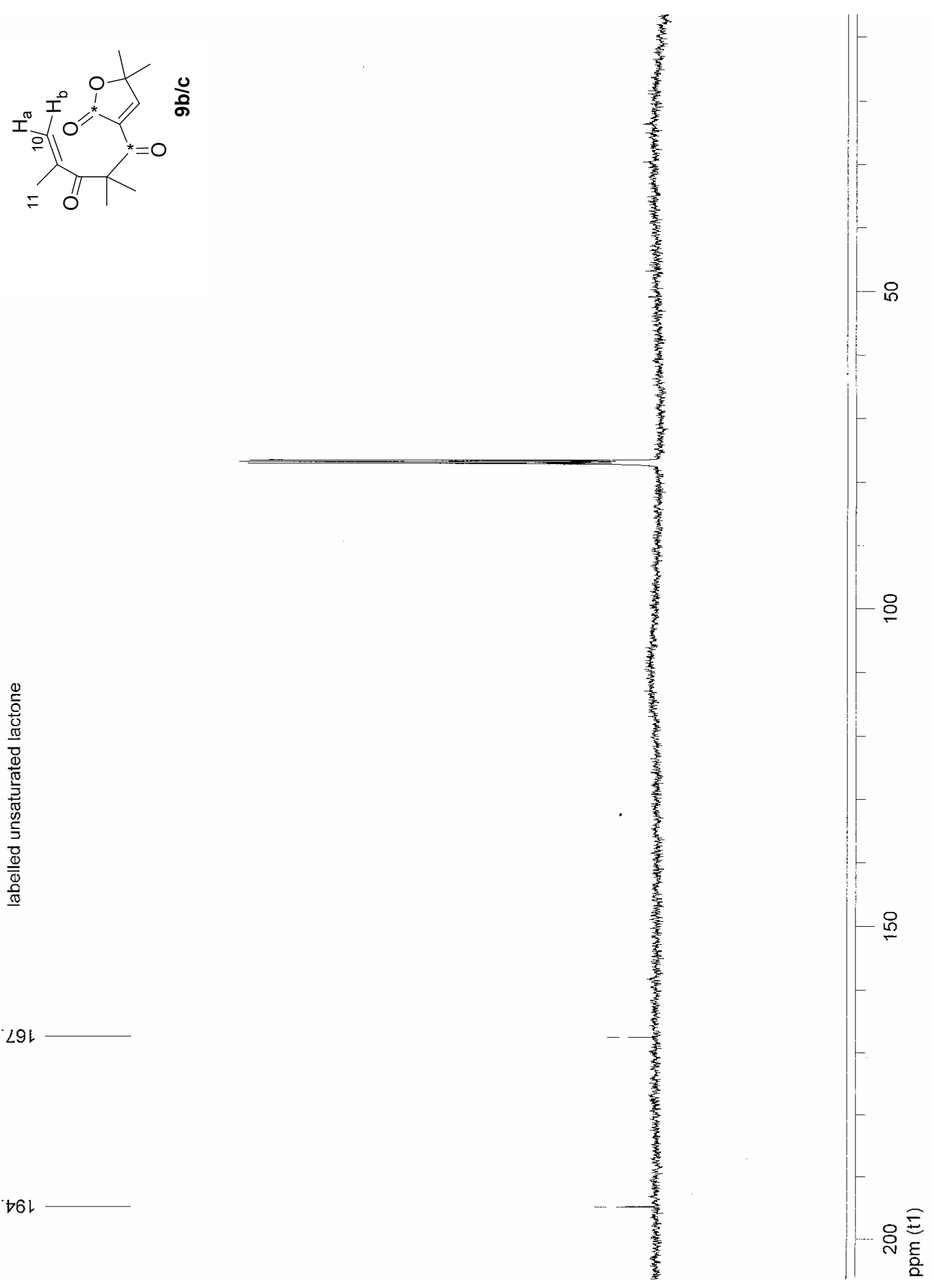

$969 \angle 9 l$

$698+61$

응

9b/c $\left.{ }^{13} \mathrm{C} \mathrm{NMR} \mathrm{(125MHz,} \mathrm{CDCl}_{3}\right)$ 

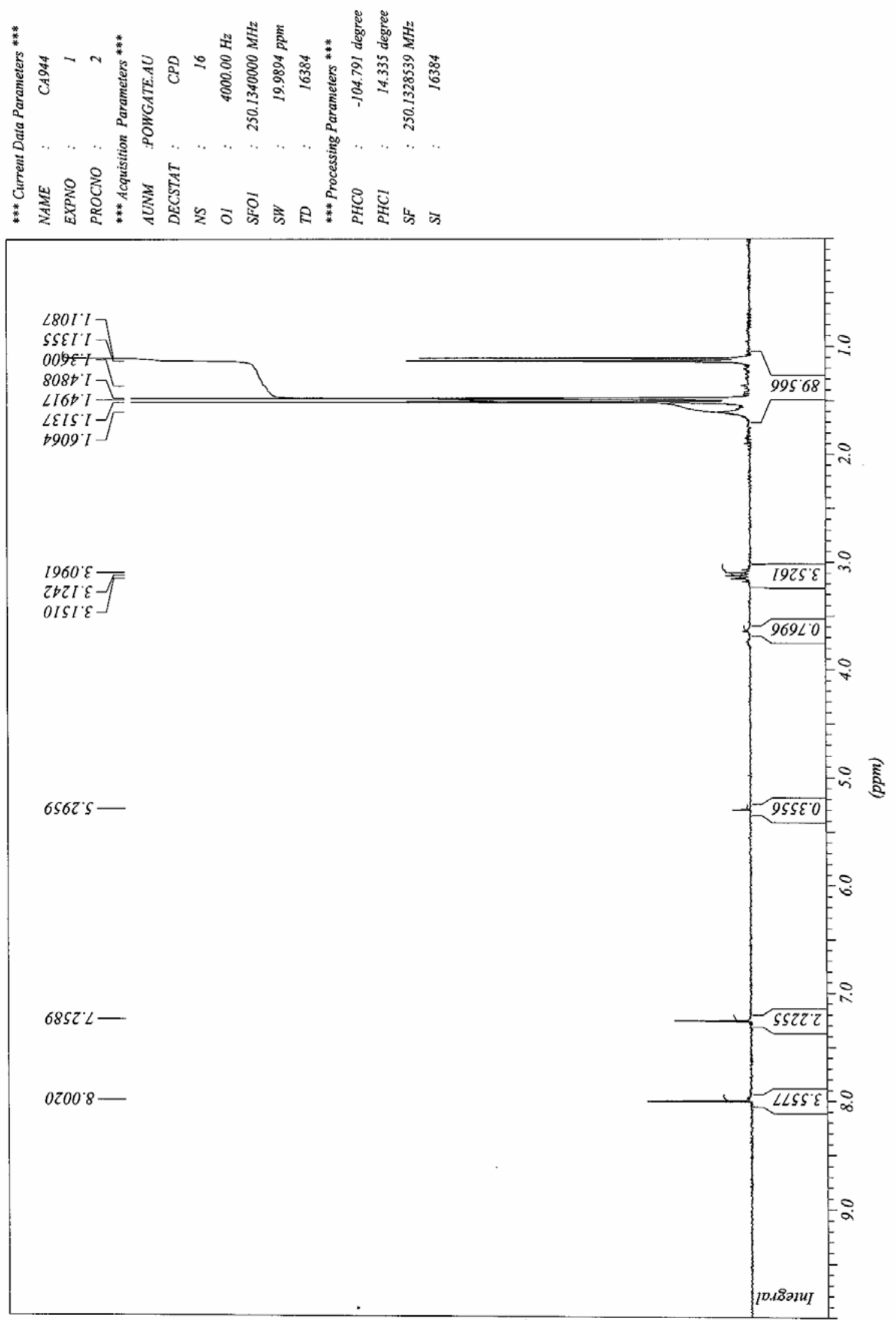

$10{ }^{1} \mathrm{H}$ NMR (400MHz, $\left.\mathrm{CDCl}_{3}\right)$ 


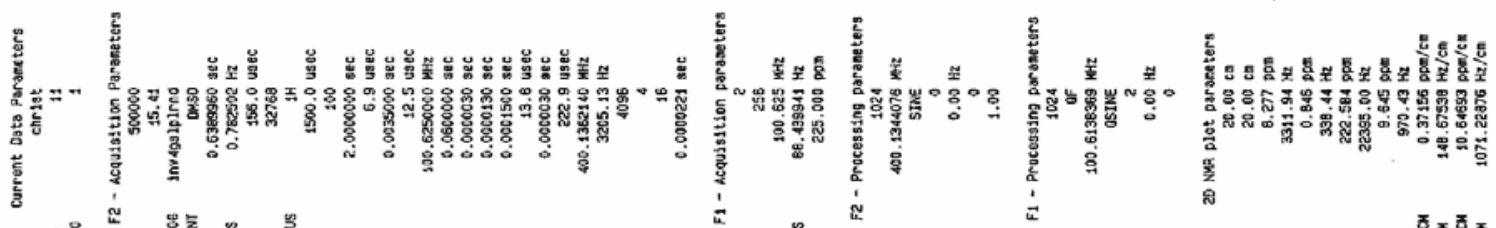

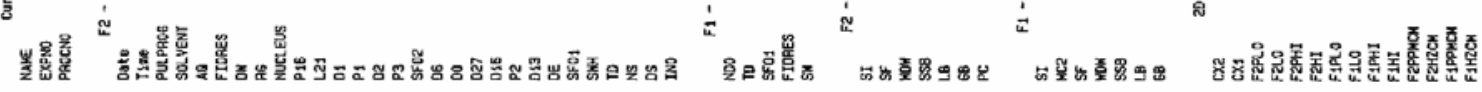
(1)

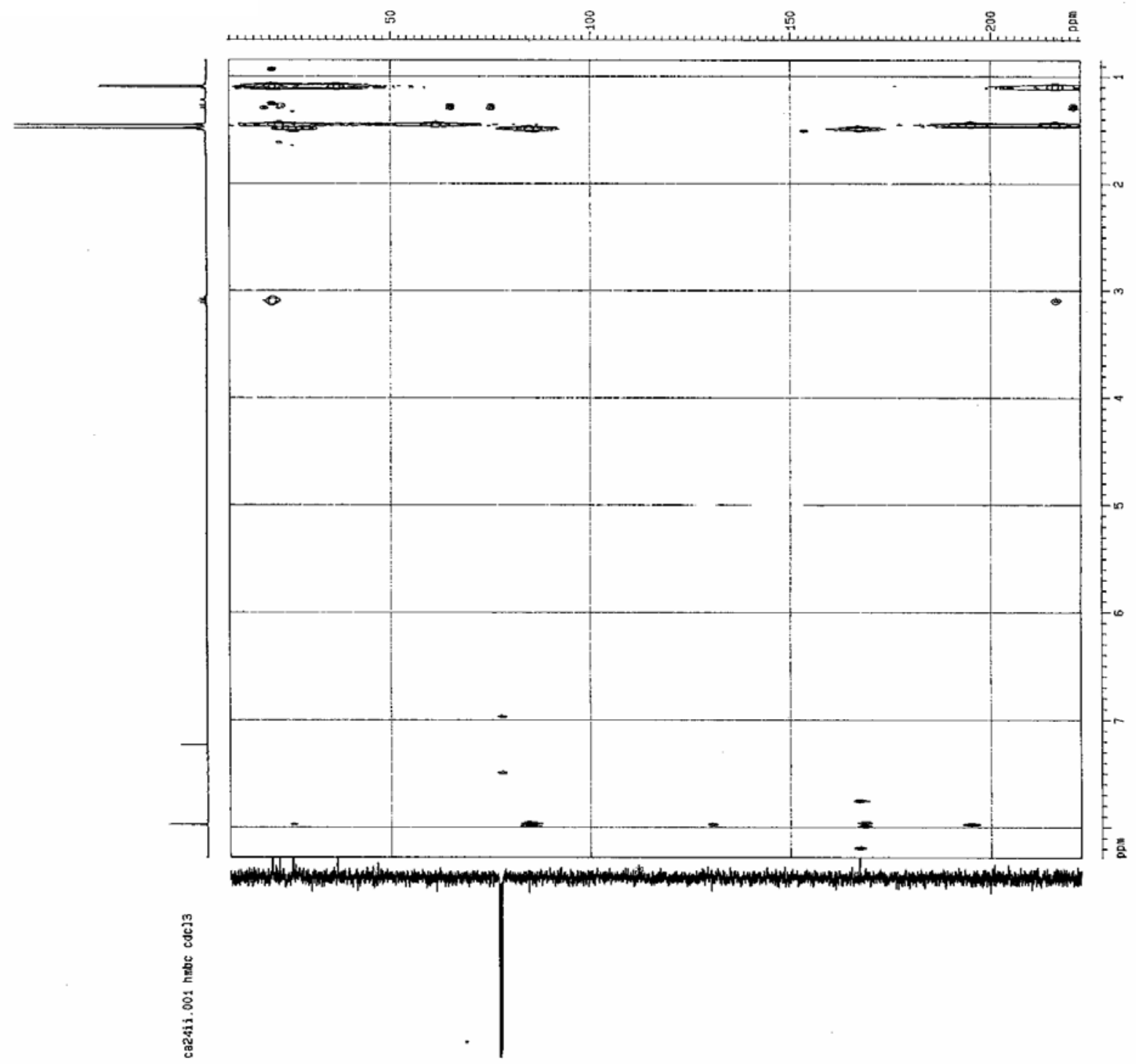

$10 \mathrm{HMBC}\left(400 \mathrm{MHz}, \mathrm{CDCl}_{3}\right)$ 


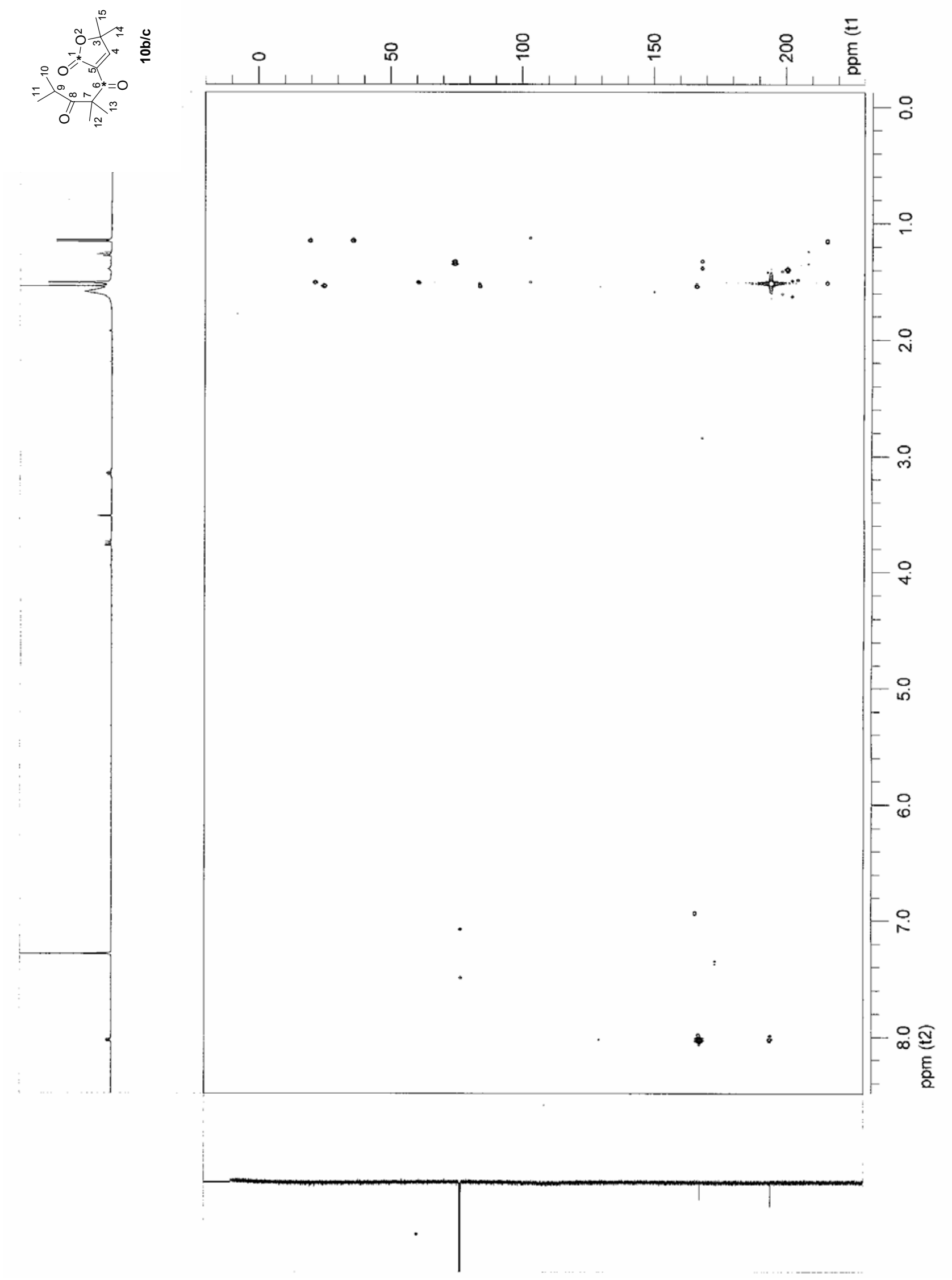

10b/c $\mathrm{HMBC}\left(400 \mathrm{MHz}, \mathrm{CDCl}_{3}\right)$ 


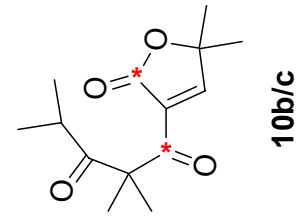

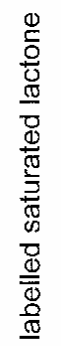

910891

$069^{\circ} \nabla 6 L$

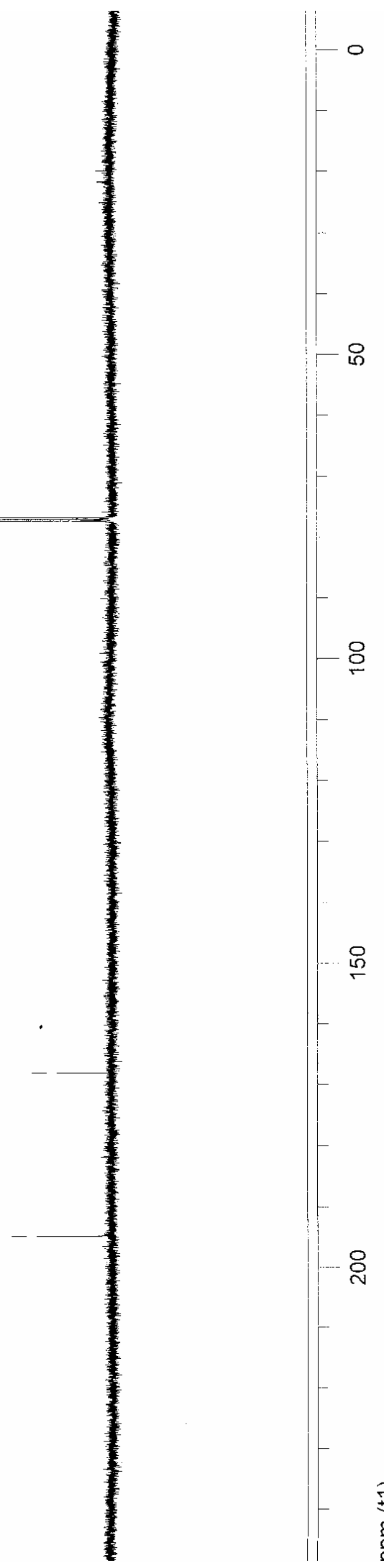

$\underset{\frac{E}{2}}{E}$

10b/c ${ }^{13} \mathrm{C}$ NMR $\left(125 \mathrm{MHz}, \mathrm{CDCl}_{3}\right)$ 

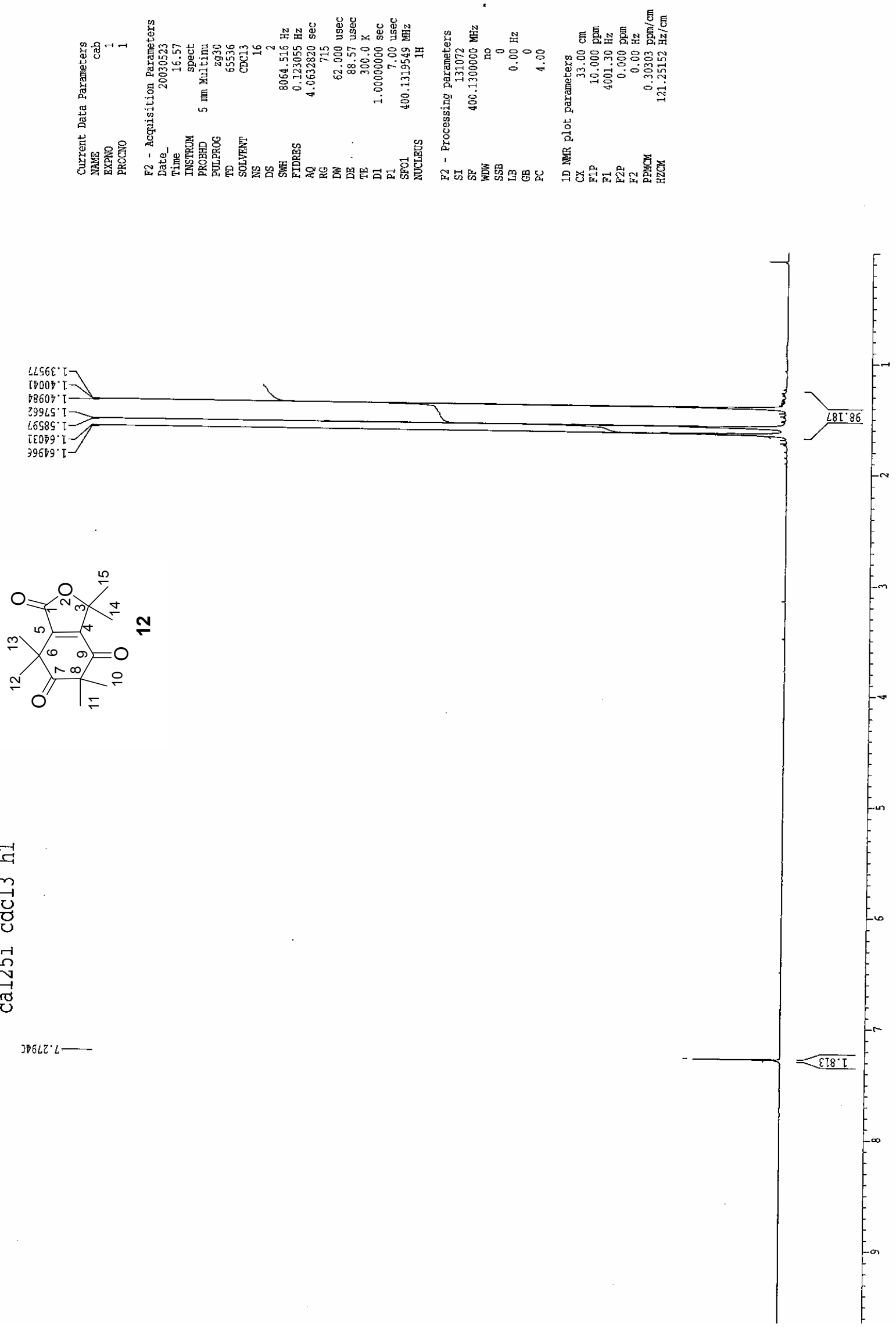

$12{ }^{1} \mathrm{H}$ NMR (400MHz, $\mathrm{CDCl}_{3}$ ) 

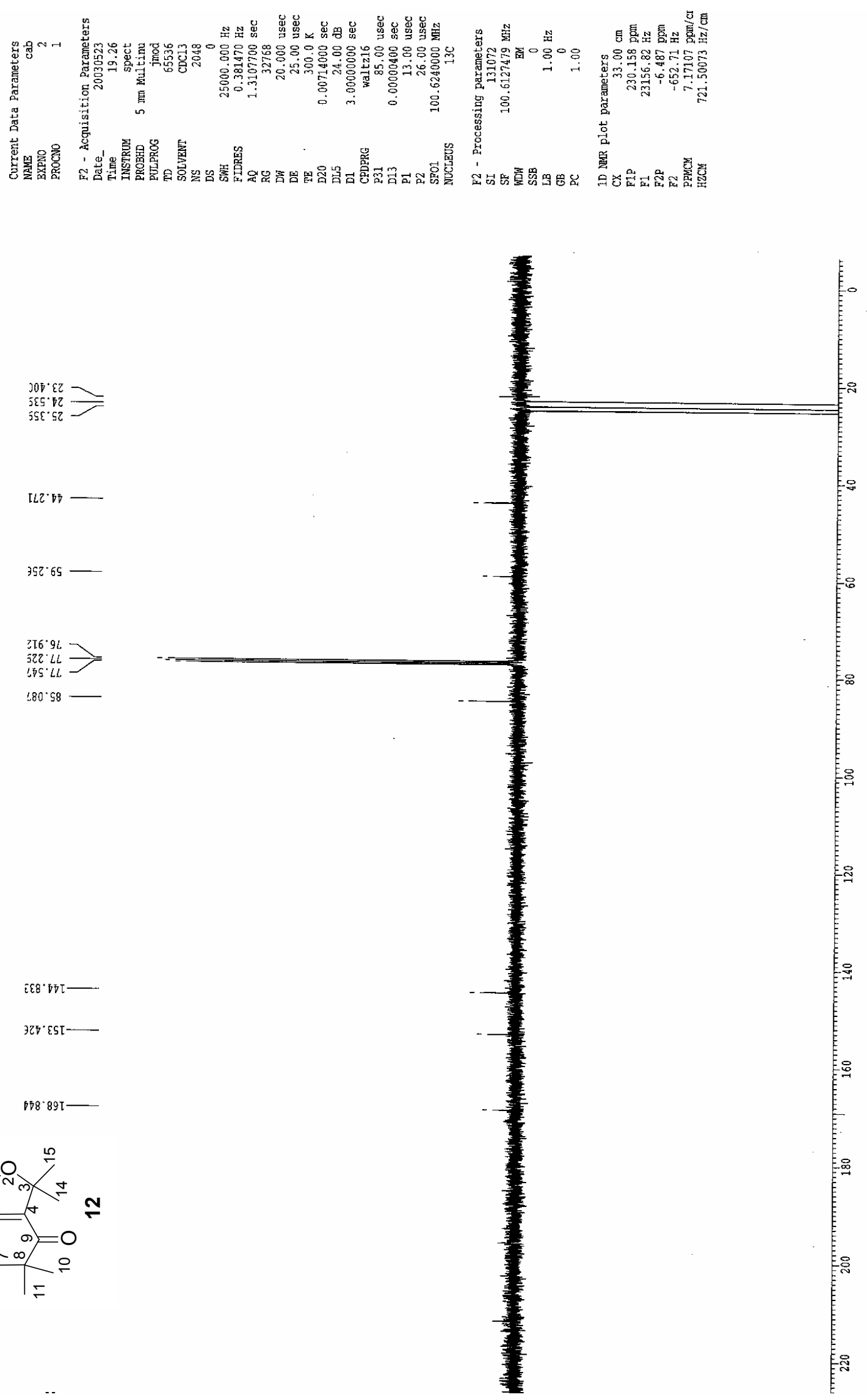

$12{ }^{13} \mathrm{C} \mathrm{NMR}\left(100.64 \mathrm{MHz}, \mathrm{CDCl}_{3}\right)$ 


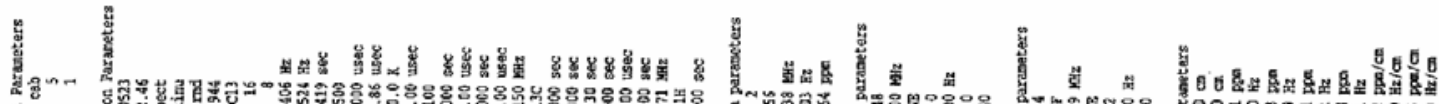

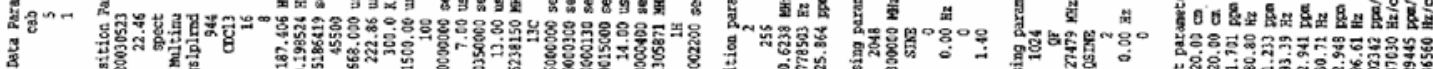

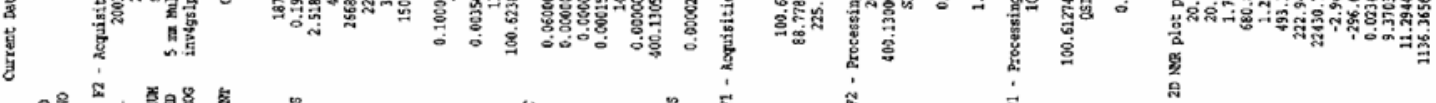

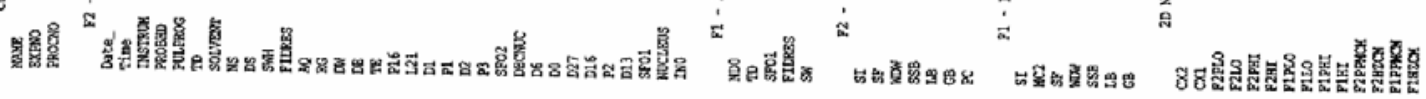

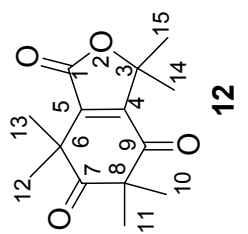

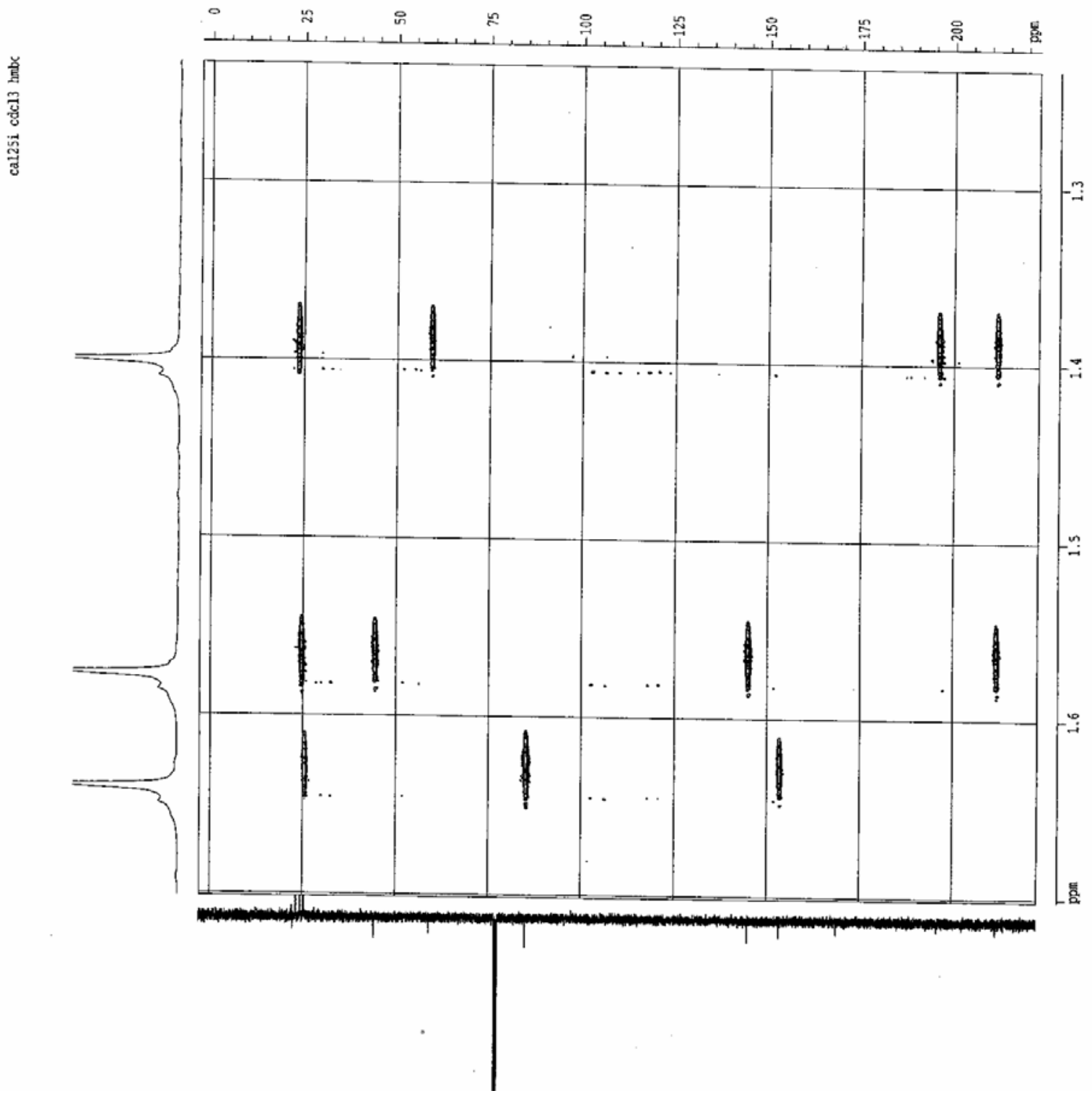

$12 \mathrm{HMBC}\left(400 \mathrm{MHz}, \mathrm{CDCl}_{3}\right)$ 


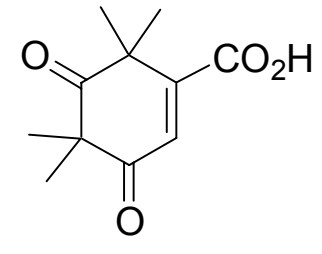

7

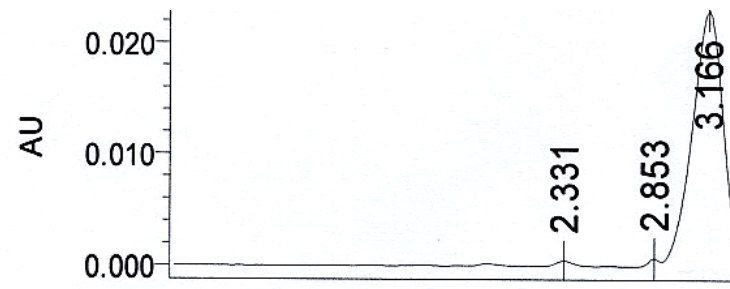



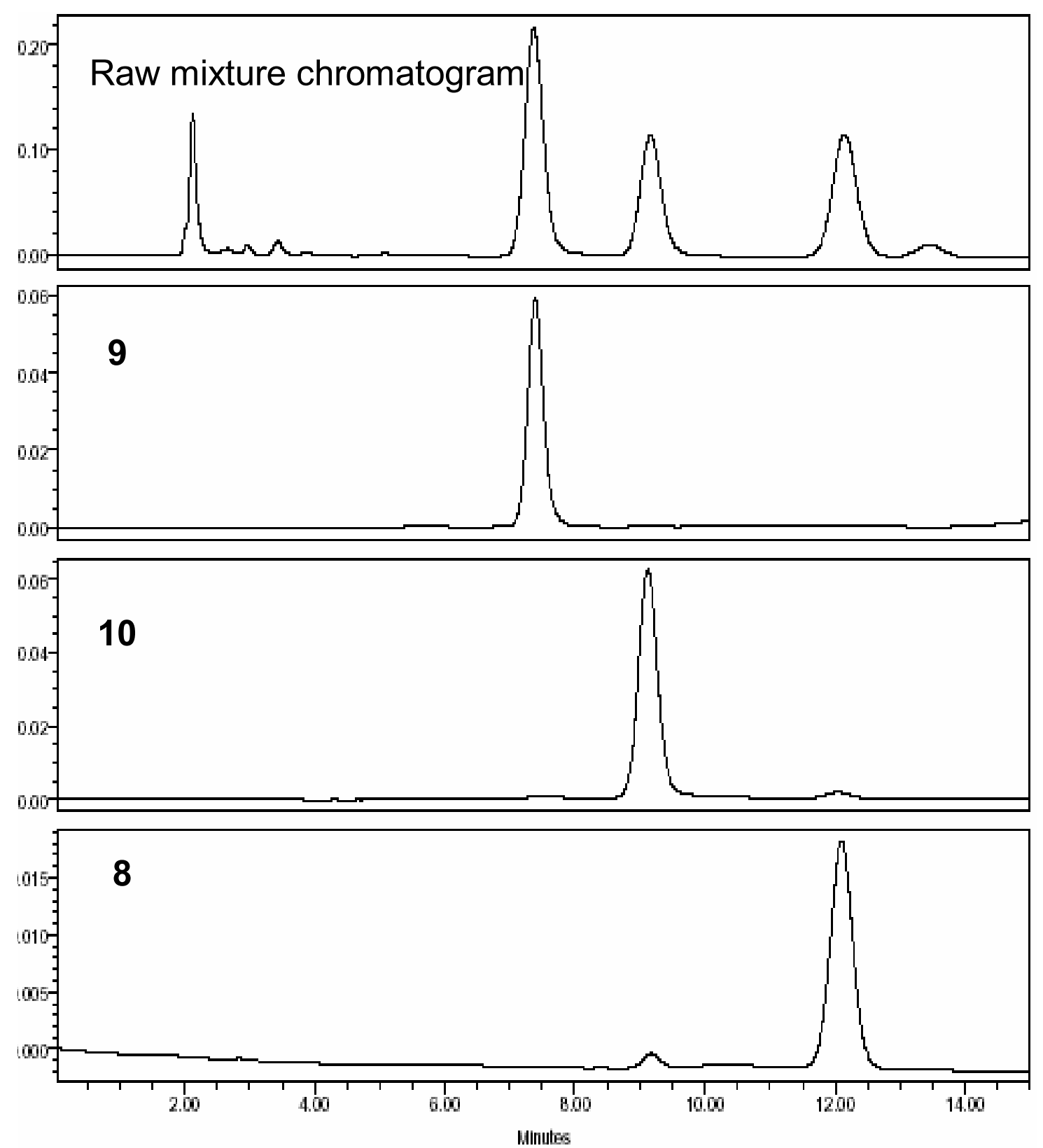